ARTICLE

\title{
Integrative oncogene-dependency mapping identifies RIT1 vulnerabilities and synergies in lung cancer
}

\author{
Athea Vichas ${ }^{1,10}$, Amanda K. Riley (10) 1,2,10, Naomi T. Nkinsi', Shriya Kamlapurkar', Phoebe C. R. Parrish (1) 1,3, \\ April Lo 1,3, Fujiko Duke4, Jennifer Chen ${ }^{4}$, Iris Fung ${ }^{4}$, Jacqueline Watson ${ }^{4}$, Matthew Rees (1) ${ }^{4}$, \\ Austin M. Gabel (10) 3,5,6,7, James D. Thomas (1) 6,7, Robert K. Bradley (10 3,6,7, John K. Lee ${ }^{1}$, Emily M. Hatch (1) 1,7, \\ Marina K. Baine ${ }^{8}$, Natasha Rekhtman ${ }^{8}$, Marc Ladanyi ${ }^{8}$, Federica Piccioni ${ }^{4,9}$ \& Alice H. Berger (1) 1,3凶
}

CRISPR-based cancer dependency maps are accelerating advances in cancer precision medicine, but adequate functional maps are limited to the most common oncogenes. To identify opportunities for therapeutic intervention in other rarer subsets of cancer, we investigate the oncogene-specific dependencies conferred by the lung cancer oncogene, RIT1. Here, genome-wide CRISPR screening in KRAS, EGFR, and RIT1-mutant isogenic lung cancer cells identifies shared and unique vulnerabilities of each oncogene. Combining this genetic data with small-molecule sensitivity profiling, we identify a unique vulnerability of RIT1mutant cells to loss of spindle assembly checkpoint regulators. Oncogenic RIT1M90I weakens the spindle assembly checkpoint and perturbs mitotic timing, resulting in sensitivity to Aurora A inhibition. In addition, we observe synergy between mutant RIT1 and activation of YAP1 in multiple models and frequent nuclear overexpression of YAP1 in human primary RIT1-mutant lung tumors. These results provide a genome-wide atlas of oncogenic RIT1 functional interactions and identify components of the RAS pathway, spindle assembly checkpoint, and Hippo/YAP1 network as candidate therapeutic targets in RIT1-mutant lung cancer.

\footnotetext{
${ }^{1}$ Human Biology Division, Fred Hutchinson Cancer Research Center, Seattle, WA, USA. ${ }^{2}$ Molecular and Cellular Biology Program, University of Washington, Seattle, WA, USA. ${ }^{3}$ Department of Genome Sciences, University of Washington, Seattle, WA, USA. ${ }^{4}$ Broad Institute of MIT \& Harvard, Cambridge, MA, USA. ${ }^{5}$ Medical Scientist Training Program, University of Washington, Seattle, WA, USA. ${ }^{6}$ Computational Biology Program, Public Health Sciences Division, Fred Hutchinson Cancer Research Center, Seattle, WA, USA. ${ }^{7}$ Basic Sciences Division, Fred Hutchinson Cancer Research Center, Seattle, WA, USA.

${ }^{8}$ Department of Pathology, Memorial Sloan Kettering Cancer Center, New York, USA. ${ }^{9}$ Present address: Merck Research Laboratories, Boston, MA, USA.

${ }^{10}$ These authors contributed equally: Athea Vichas, Amanda K. Riley. ${ }^{凶}$ email: ahberger@fredhutch.org
} 
S omatic mutations that activate EGFR/RAS pathway signaling are a hallmark of lung adenocarcinoma, occurring in more than $75 \%$ of tumors ${ }^{1}$. Oncogenes in the EGFR/RAS pathway display 'oncogene addiction', a tumor-specific reliance on sustained cell signaling for cell survival, and consequently these mutated oncogenes represent powerful drug targets for lung cancer therapy ${ }^{2}$. Several of the mutated genes in this pathway are clinically targeted to improve outcomes for lung cancer patients. For example, somatic mutations in EGFR underlie sensitivity to EGFR inhibitors erlotinib and osimertinib ${ }^{3,4}$, and chromosomal rearrangements involving $A L K$ underlie sensitivity to crizotinib ${ }^{5}$ and other ALK inhibitors.

Recently, we connected RIT1 to the EGFR/RAS protumorigenic signaling network $\mathrm{k}^{6-8}$. RIT1 is mutated in $2 \%$ and amplified in $14 \%$ of lung adenocarcinomas ${ }^{6,7}$. RIT1 encodes a ubiquitously expressed RAS-family GTPase protein ${ }^{9,10}$. Mutations in RIT1 are mutually exclusive with mutations in KRAS, $E G F R, A L K, M E T$, and other driver oncogenes in lung adenocarcinoma, suggesting that RIT1 may drive EGFR/RAS pathway activation in RIT1-mutant tumors ${ }^{6}$. Consistent with this idea, mutant RIT1 can transform NIH3T3 fibroblasts and confer resistance to EGFR inhibition in EGFR-mutant lung cancer cells $^{7,8}$. Mutant RIT1 has been identified in a patient with acquired resistance to ALK inhibition ${ }^{11}$. Somatic RIT1 mutations also occur in myeloid malignancies ${ }^{12}$, and focal RIT1 amplifications are observed in uterine carcinosarcoma ${ }^{13}$. Because RIT1 mutations are mutually exclusive with other driver alterations in lung adenocarcinoma ${ }^{6}$, patients with RIT1-mutant lung tumors have limited therapeutic options of standard chemo- and immuno-therapy regimens but no targeted therapies. Beyond cancer, germline mutations in RIT1 are found in patients with the RAS-opathy Noonan syndrome (NS) ${ }^{14}$.

Both RIT1 protein abundance ${ }^{15}$ and GTP binding ${ }^{16}$ appear to be central to its oncogenic function. RIT1 mutations disrupt RIT1's negative regulation by the ubiquitin adaptor protein LZTR1, leading to increased RIT1 protein abundance. Cancerand NS-associated variants also display decreased GTP hydrolysis, increased nucleotide exchange, or increased GTP binding ${ }^{15,16}$, and the exact contribution of GTP binding versus protein abundance to RIT1's oncogenic function remains to be determined. In both cancer and NS, a similar spectrum of RIT1 missense and in-frame insertion/deletion mutations is observed, with the majority occurring in the switch II domain of the protein. Mutation at methionine 90 (M90I) is recurrent, but the spectrum of mutations is relatively diverse and the majority have been shown to confer the same cellular transformation capability ${ }^{7}$ and loss of LZTR1 binding ${ }^{15}$. Beyond these observations, relatively little is known about the specific mechanism of action of RIT1, how it differs from KRAS, and what proteins are critical to induce its oncogenic function. Further understanding the cellular consequences of oncogenic RIT1 mutations could open up strategies for therapeutic intervention in RIT1-mutant cancers and Noonan syndrome.

In this work, to investigate the structure of the EGFR/RAS/ RIT1 signaling network and identify therapeutic targets in lung cancer, we perform genome-wide CRISPR screens in isogenic PC9 cells where drug resistance is potently conferred by each expressed oncogene. We find that the dependencies identified broadly confirm the expected pathway hierarchy but also identify key differences that highlight the importance of genotype-guided treatment stratification. A key difference we identify is in sensitivity to mitotic perturbation; RIT1- and KRAS-mutant cells differ in their sensitivity to Aurora kinase inhibitors due to a role of RIT1 $^{\mathrm{M} 90 \mathrm{I}}$ in the spindle assembly checkpoint. Furthermore, we identify YAP1 activation as a key cooperating event in RIT1mutant lung cancer. In addition, we identify many other candidate EGFR-, KRAS-, and RIT1 dependencies that should be further explored as drug targets for lung cancer therapy. This study expands our knowledge of EGFR/RAS signaling in lung cancer and provides a genome-wide discovery of factors that cooperate with or antagonize oncogenic RIT1.

\section{Results}

Context-specific differences in RIT1- and KRAS-stimulated ERK activity. Our previous work found that cancer- and Noonanassociated RIT1 variants such as RIT1 ${ }^{\text {M90I }}$ can transform NIH3T3 fibroblasts to a phenotype reminiscent of RAS-transformed cells ${ }^{7}$. However, when we examined cellular signaling by Western blot in the same cells, we discovered that oncogenic RIT1 variants fail to promote AKT, MEK, or ERK phosphorylation, whereas both $\mathrm{KRAS}^{\mathrm{G} 12 \mathrm{~V}}$ and HRAS ${ }^{\mathrm{G} 12 \mathrm{~V}}$ induce marked increases in MEK/ERK phosphorylation (Fig. 1a). Prior data showed that RIT1 variants are capable of robustly activating AKT, MEK, and ERK in PC6 pheochromocytoma cells ${ }^{7}$, but more subtly induce MEK/ERK phosphorylation in murine embryonic fibroblasts ${ }^{15}$, so we examined additional models to see if RIT1 ${ }^{\mathrm{M} 90 \mathrm{I}}$ function varies in a cell-type dependent manner. Expression of RIT1 ${ }^{\mathrm{M} 90 \mathrm{I}}$ in two different human lung epithelial cell lines, SALE or AALE ${ }^{17}$, did increase MEK phosphorylation, but the degree of stimulation differed between the two cell lines despite similar RIT1 ${ }^{\mathrm{M} 90 \mathrm{I}}$ levels (Supplementary Fig. 1a). AKT phosphorylation was weakly induced in SALE cells only. These data suggest that context-dependent factors determine RIT1's ability to stimulate MEK and AKT phosphorylation.

We previously demonstrated that RIT1 ${ }^{\text {M90I }}$ and other RIT1 variants, as well as KRAS G12V, confer resistance to EGFR targeted therapy in EGFR-mutant lung cancer cells ${ }^{8}$. Expression of either RIT1 ${ }^{\text {M90I }}$ or KRASG12V in PC9 EGFR-mutant lung adenocarcinoma cells renders them resistant to the EGFR inhibitors erlotinib (Fig. 1b) or osimertinib (Fig. 1c), conferring an almost 1000-fold decrease in drug sensitivity. Despite similar degrees of erlotinib- and osimertinib resistance in PC9-RIT1 ${ }^{\text {M90I }}$ and PC9KRASG12V cells, induction of ERK phosphorylation in RIT1mutant cells was absent to low, while KRAS-mutant cells showed marked rescue of phosphorylated ERK levels (Fig. 1d). Phosphorylation of AKT was induced similarly in RIT1- and KRASmutant cells (Fig. 1d). We confirmed that resistance conferred by $\mathrm{RIT1}^{\mathrm{M} 90 \mathrm{I}}$ required continued expression of $\mathrm{RIT1}^{\mathrm{M} 90 \mathrm{I}}$ because erlotinib or osimertinib resistance could be reversed by knockout of RIT1 using CRISPR-Cas9 technology (Fig. 1e, Supplementary Fig. 1b-d). These data indicate that stimulation of ERK phosphorylation by RIT1 is cell-type dependent and may involve distinct effectors than KRAS. This finding prompted us to develop a systematic approach to map the differing cellular consequences of oncogenic RIT1 and KRAS activation.

\section{A genome-scale platform for identification of oncogene-} specific dependencies in lung cancer. Genome-scale CRISPR screens have been used to identify genetic dependencies of oncogenes in cancer, notably $K R A S^{18-21}$. Comparative analyses of dependencies in mutant versus wild-type cell lines such as those from the Broad Institute Dependency $\mathrm{Map}^{22}$ are powerful resources for the discovery of oncogene vulnerabilities. However, the vast majority of mutated cancer genes occur in a relatively low fraction of tumors and cell lines. Indeed, only one lung adenocarcinoma cell line, NCI-H2110, has been identified with a canonical RIT1 $^{\mathrm{M} 90 \mathrm{I}}$ mutation ${ }^{7}$. An alternative strategy is CRISPR screens of isogenic cell models ${ }^{20,23}$ in which introduced oncogenes confer a selectable phenotype to the cells. Because RIT1 variants can confer resistance to erlotinib in PC9 cells, an EGFRmutant lung cancer cell line $e^{8}$, this drug resistance phenotype could provide a powerful screening system to probe the 
a

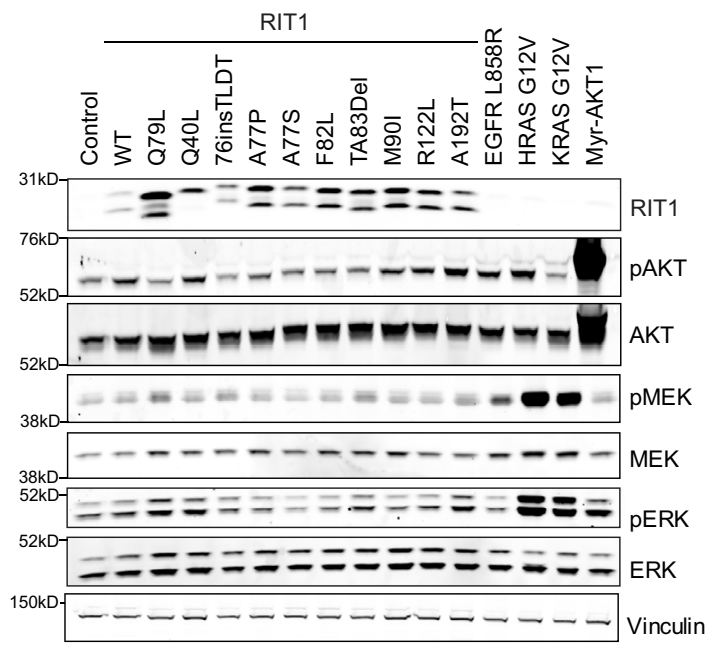

b

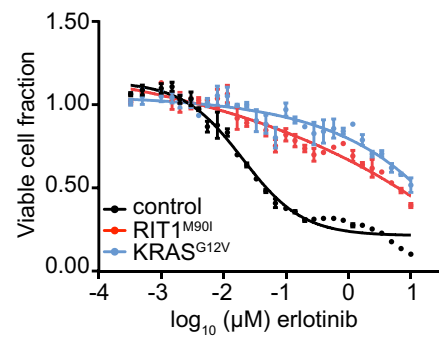

d

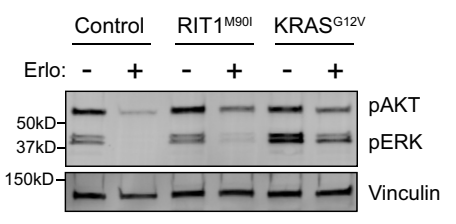

C
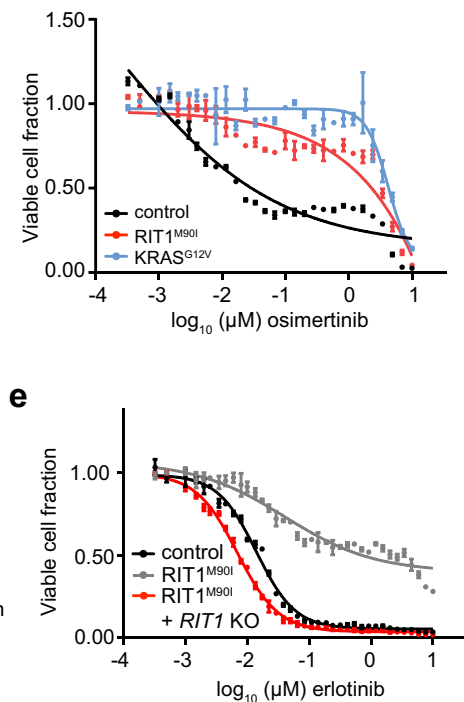

Fig. 1 RIT1 ${ }^{\text {M90I }}$ and KRAS ${ }^{\mathbf{G 1 2 V}}$ promote resistance to EGFR tyrosine kinase inhibitors. a Western blot of lysates from NIH3T3 cells stably expressing a

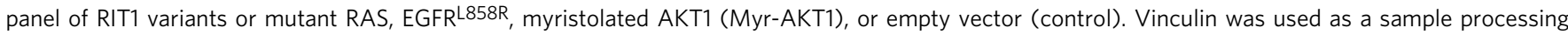
control. pAKT, phosphorylated Ser473 AKT1/2/3. pERK, phosphorylated Thr202/Tyr204 ERK1/2. pMEK, phosphorylated Ser217/221 MEK1/2. b Dose-response curve of 96-hour erlotinib treatment in isogenic PC9-Cas9 cells expressing the indicated oncogene or Firefly luciferase (control). CellTiterGlo was used to quantify viable cell number and viable cell fraction determined by normalization to DMSO control. Data shown are the mean \pm s.e. m. of two technical replicates. c Dose-response curve of osimertinib, performed as in (b). Data shown are the mean \pm s.e.m. of two technical replicates. $\mathbf{d}$ Western blot of lysates from cells shown in (b, c), cultured in the absence or presence of 500 nM erlotinib for $72 \mathrm{~h}$. pAKT, phosphorylated Ser473 AKT1/ 2/3. pERK, phosphorylated Thr202/Tyr204 ERK1/2. Vinculin was used as a loading control. Blot representative of $n=2$ independent experiments. e Dose-response curve of $96 \mathrm{~h}$ erlotinib treatment in clonal RIT1 knockout (KO) cells compared to parental PC9-Cas9-RIT1M90I cells or control PC9 cells. Data generated and presented as in (b). Data shown are the mean \pm s.d. of two technical replicates. Unless otherwise indicated, all data are representative results from $n=2$ independent experiments. Source data are provided as a Source Data file.

requirements for RIT1 function in a highly controlled and robust system.

As a proof of concept, we tested whether small-molecule inhibition of downstream signaling components of the EGFR/ RAS pathway could overcome RIT1 ${ }^{\text {M90I }}$-induced erlotinib resistance in PC9 cells. We ectopically expressed RIT1 ${ }^{\mathrm{M} 90 \mathrm{I}}$ and $\mathrm{KRAS}^{\mathrm{G} 12 \mathrm{~V}}$ in PC9 cells using lentiviral transduction and then cultured the cells in the presence of erlotinib or torin1, a smallmolecule inhibitor of mTOR. Co-treatment with torin 1 partially re-sensitized cells to erlotinib, demonstrating that both RIT1 ${ }^{\mathrm{M} 90 \mathrm{I}}$ and KRAS ${ }^{\mathrm{G} 12 \mathrm{~V}}$ likely act upstream of mTOR to induce erlotinib resistance (Fig. 2a).

To systematically define the factors required for RIT1 ${ }^{\text {M90I }}$ - and $\mathrm{KRAS}^{\mathrm{G} 12 \mathrm{~V}}$-driven drug resistance we developed a pooled genome-wide knockout approach in drug-resistant PC9 cells (Fig. 2b). Isogenic pools of PC9 cells were generated expressing Cas9 and either a Firefly luciferase vector negative control (control), RIT1 ${ }^{\mathrm{M} 90 \mathrm{I}}$, KRAS ${ }^{\mathrm{G} 12 \mathrm{~V}}$, or $\mathrm{EGFR}^{\mathrm{T} 790 \mathrm{M} / \mathrm{L} 858 \mathrm{R} \text {, an }}$ erlotinib-resistant mutant of EGFR used as a positive control (Supplementary Fig. 2a). Cas9 activity was confirmed by a sgGFPGFP flow cytometry assay (Supplementary Fig. 2b, c). Long-term erlotinib resistance was assessed by culturing cells in the presence of $50 \mathrm{nM}$ erlotinib for 22 days (Fig. 2c). Whereas cells expressing $\mathrm{RIT1}^{\mathrm{M} 90 \mathrm{I}}, \mathrm{KRAS}^{\mathrm{G} 12 \mathrm{~V}}$, or $\mathrm{EGFR}^{\mathrm{T} 790 \mathrm{M} / \mathrm{L} 858 \mathrm{R}}$ all proliferated at a similar rate in erlotinib, control PC9 cells failed to expand in erlotinib (Fig. 2c).

To identify genetic dependencies required for PC9-Cas9RIT1 $^{\text {M90I, PC9-Cas9-KRASG12V, and PC9-Cas9-EGFR }}{ }^{\text {T790M/ }}$ L858R cell proliferation in erlotinib, we used the Brunello CRISPR library consisting of 1000 non-targeting guides and 76,441 guides targeting 19,114 human genes ${ }^{24}$. Cells were transduced, selected with puromycin, and split to erlotinib or vehicle (DMSO) conditions, then maintained in erlotinib or DMSO for approximately 12 population doublings (Supplementary Fig. 2d). The change in abundance of each sgRNA was determined by sequencing and comparing the endpoint sgRNA abundance to the initial sgRNA abundance in the plasmid library (Supplementary Data 1), which was highly correlated with early time point (ETP) replicates taken just after lentiviral transduction and puromycin selection. Replicate screens were well-correlated $\left(R^{2}\right.$ range 0.58-0.94, Supplementary Fig. 2e) and exhibited expected lethality of known essential genes with a median strictly standardized mean difference (SSMD) of -3.9 (Supplementary Fig. 2f, Supplementary Data 1). To enable quantitative comparisons across screens, we adapted previously established methods ${ }^{22}$ to compute normalized CRISPR scores (CS), scaling the data such that the median CS of all sgRNAs targeting known essential genes is -1 and the median CS of those targeting known nonessential genes is 0 (Methods, Supplementary Data 2). As expected, expressed genes were, on average, more essential than nonexpressed genes (Supplementary Fig. 2g). Importantly, as expected, sgRNAs targeting KRAS or RIT1 were negatively selected in PC9-Cas9-KRASG12V and PC9-Cas9-RIT1 ${ }^{\text {M90I }}$ cells, respectively, in erlotinib (Fig. 2d), validating the requirement of each oncogene for cell survival. As predicted, control PC9 cells are already dependent on EGFR for survival, so PC9-Cas9 cells expressing the erlotinib-resistant $\mathrm{EGFR}^{\mathrm{T} 790 \mathrm{M} / \mathrm{L} 858 \mathrm{R}}$ variant also remain dependent on EGFR in both erlotinib-treated and vehicletreated conditions (Fig. 2d).

The concept of synthetic lethality originally referred to the phenomenon in which two genetic mutations cause a lethal phenotype whereas either mutation alone is tolerated. In cancer 
a
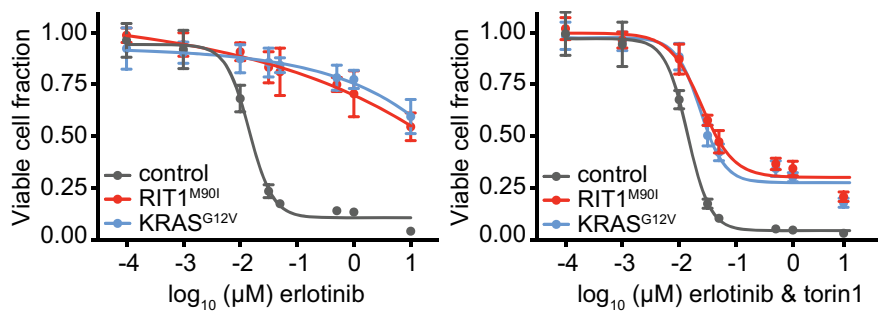

b

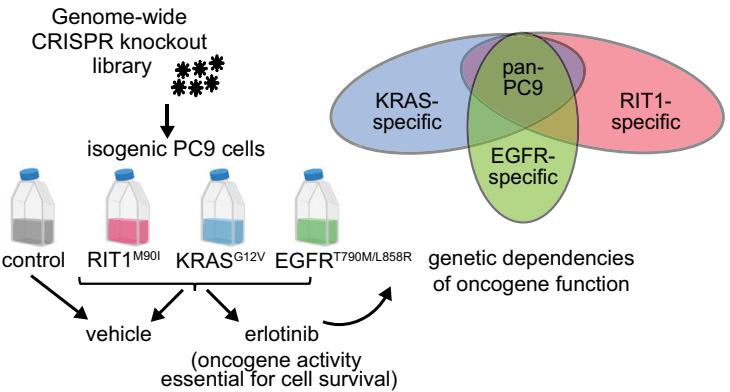

C

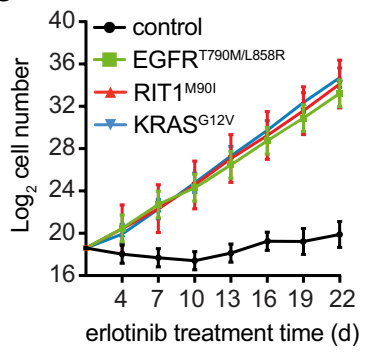

d

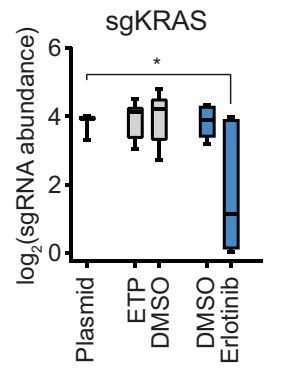

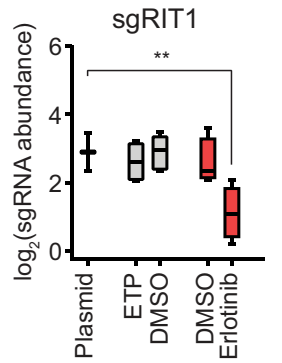

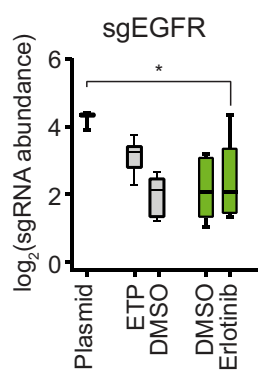

$\square$ plasmid

$\square$ control

$\square$ KRAS $^{\mathrm{G} 12 \mathrm{~V}}$

$\square$ RIT1 ${ }^{\text {M90 }}$

$\square$ EGFR ${ }^{\mathrm{T} 790 \mathrm{M} / \mathrm{L} 858 \mathrm{R}}$

Fig. 2 Building an integrative oncogene-dependency map of EGFR ${ }^{\text {T790M/L858R, }}$ KRAS ${ }^{\mathbf{G 1 2 V}}$, and RIT1M90I. a 96-hour dose-response of isogenic PC9 cells to erlotinib alone (left panel) or to a 1:1 molar ratio of erlotinib and torin1 (right panel). Fraction of viable cells was determined using CellTiterGlo and normalized to the average value in DMSO treated cells. Data shown are the mean \pm s.d. of 8 technical replicates. $\mathbf{b}$ Schematic of the genome-wide CRISPRCas9 screens performed in isogenic PC9 cells. Created with BioRender.com. c, Proliferation rates of the PC9-Cas9 isogenic cells used for genome-wide screening in $50 \mathrm{nM}$ erlotinib. Data shown are the mean $\pm 95 \%$ confidence interval for two replicates per cell line. $\mathbf{d}$ Box plot showing sgRNA abundance ( $\log _{2}$ reads per million) of sgRNAs targeting each indicated gene. Abundance in the plasmid library, early time point (ETP), or after 12 population doublings in vehicle (DMSO) or erlotinib was determined by PCR and Illumina sequencing. Box plots show the median (center line), first and third quartiles (box edges), and the min and max range (whiskers) of replicates. ${ }^{\star} p=0.022$ (sgKRAS), ${ }^{\star \star} p=0.009$ (sgRIT1), * $p=0.004$ (sgEGFR) calculated by one-sided permutation testing using MAGeCK. For control PC9 cells ETP and DMSO, $n=3$ biological replicates. For oncogene-expressing PC9 cells DMSO and erlotinib, $n=2$ biological replicates. Source data are provided as a Source Data file.

biology, the concept has been adapted to identify oncogene dependencies that might be leveraged for cancer therapy ${ }^{25-27}$. Such vulnerabilities could be directly related to the oncogene's reliance on downstream effectors and pathways, or they might be created via indirect rewiring of cell phenotype. Here we define both baseline synthetic lethal knockouts and oncogene dependencies. We consider baseline synthetic lethal genes to be those whose knockout is lethal in the baseline DMSO treatment condition of oncogene-expressing cells but not vector control PC9 cells (Supplementary Data 3). In contrast, we define oncogene dependencies as those genes that are specifically required for the phenotype directly attributed to the oncogene-in this case, cell survival upon EGFR inhibition (Supplementary Data 4). It is reasonable that either baseline synthetic lethal or oncogene dependencies could be useful for therapeutic development in oncology, but here we focus specifically on oncogene dependencies because these are definitively linked to the assay phenotype. We expect many of these to also be essential for tumorigenesis induced by each oncogene (see below).

No evidence of genetic interaction between KRAS and RIT1. Next, we sought to understand whether RIT1 and KRAS were required for drug resistance induced by each other. To this end, we evaluated whether knockout of RIT1 underwent selection in

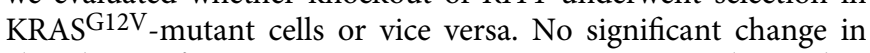
abundance of RIT1- or KRAS-targeting sgRNAs was observed in the other cell line (Supplementary Fig. 2h, i), suggesting that RIT1 and KRAS are not required for drug resistance conferred by the other gene. A similar result was seen for RIT1 and KRAS sgRNAs

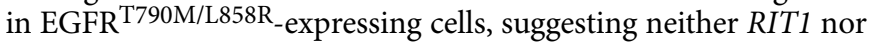

$K R A S$ is required for drug resistance driven by EGFR ${ }^{T 790 M / L 858 R}$. Given the well-known role of RAS activation downstream of $\mathrm{EGFR}^{28}$, this result is surprising. However, it is thought that activation of PI3K in EGFR-mutant cells is sufficient for cell survival in erlotinib ${ }^{29}$. It is also possible that expression of KRAS paralogs HRAS and NRAS maintain RAS activation in the absence of KRAS, since paralog redundancy is increasingly recognized to influence CRISPR knockout phenotypes ${ }^{30,31}$.

Acquired KRAS mutations can drive EGFR inhibitor resistance in patients but are rarely observed ${ }^{32}$. The low rate of acquired KRAS mutations may be due to the mutual exclusivity of KRAS and EGFR mutations in primary lung adenocarcinoma ${ }^{6,33}$. Work in mouse models has shown that co-expression of mutant KRAS and EGFR is detrimental to cancer cells, leading to negative selection of cells co-expressing both oncogenes ${ }^{34}$. If true, knockout of EGFR should be positively selected in KRAS-mutant cells. In support of this model, KRAS $\mathrm{G}^{2} \mathrm{~V}_{\text {-mutant }}$ cells better tolerated loss of EGFR in erlotinib, maintaining high abundance of EGFR sgRNAs, in contrast to control PC9 cells or EGFR- or RIT1-mutant cells (Supplementary Fig. 2j).

Integrative analysis of isogenic CRISPR screen data reveals oncogene-dependent biology. To identify significantly enriched and depleted gene knockouts, we applied MAGeCK ${ }^{35}$, which identified an average of 64 positively selected gene knockouts and 1369 essential genes per cell line in erlotinib $(|\mathrm{CS}|>0.5$ and $p<$ 0.05; Supplementary Data 2). Of these, 813 were shared across all three isogenic cell lines in erlotinib whereas 238, 209, and 157 were unique to EGFR, KRAS, and RIT1-mutant cells, respectively (Fig. 3a, Supplementary Data 4). As expected from prior 
a

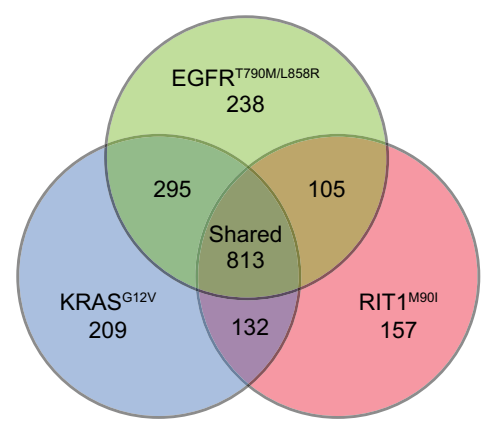

b

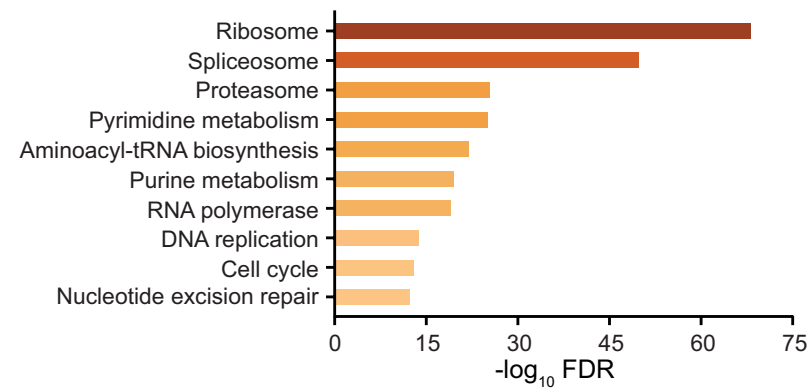

C

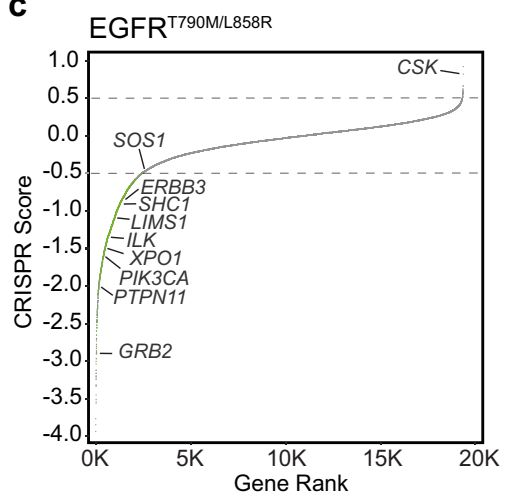

f

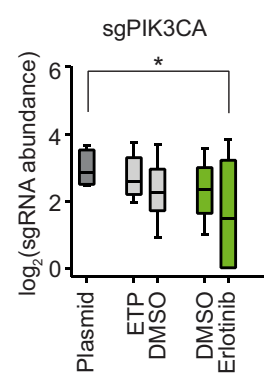

I sgIGF1R $\mathbf{m}$

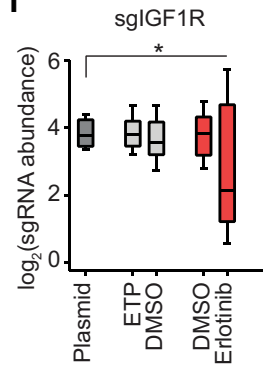

g

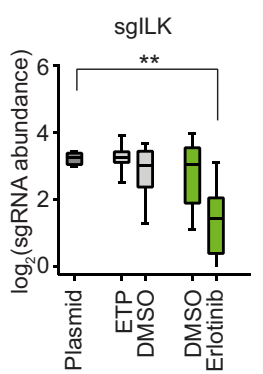

$m$

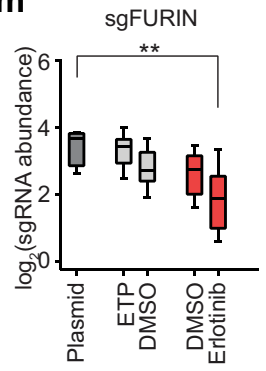

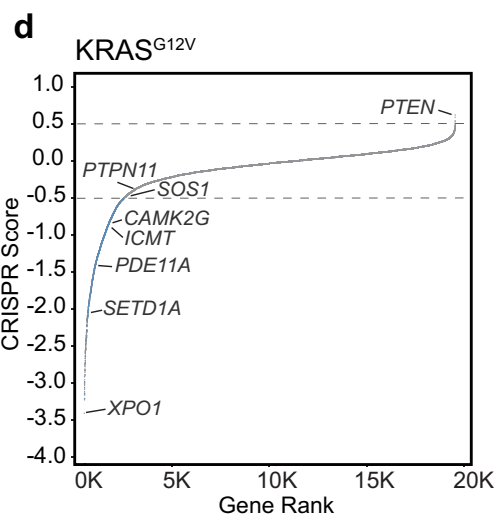

h

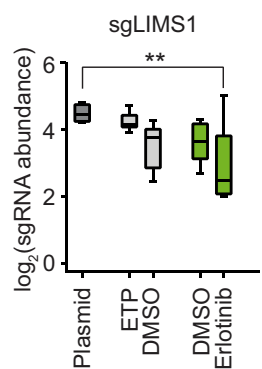

n

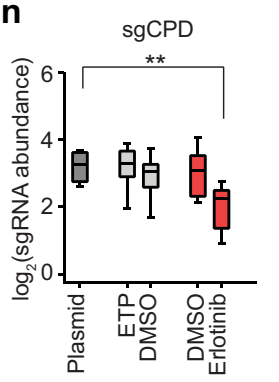

i

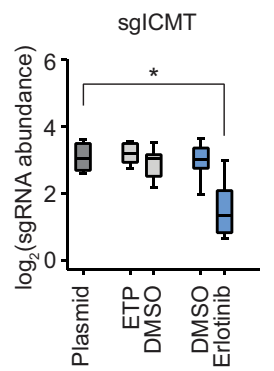

$\square$ plasmid

$\square$ control

$\square$ KRAS $^{\mathrm{G} 12 \mathrm{~V}}$

$\square$ RIT1 ${ }^{\text {M90I }}$

$\square$ EGFR $^{\mathrm{T} 790 \mathrm{M} / \mathrm{L} 858 \mathrm{R}}$

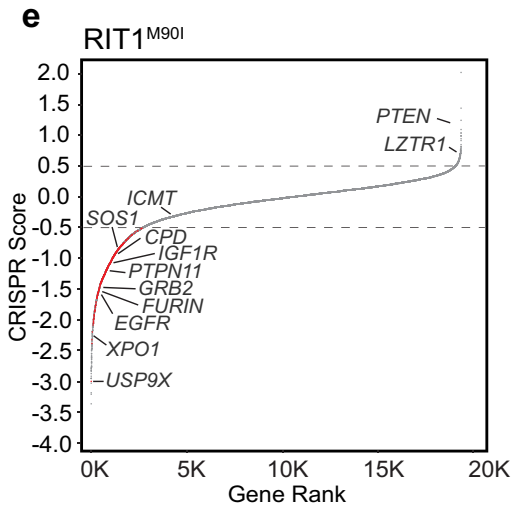

j

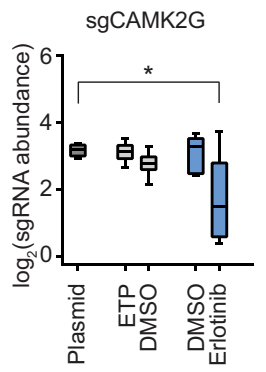

k studies $^{36}$, pan-essential genes were significantly enriched for spliceosomal, ribosomal, and proteasomal genes (Fig. 3b).

Integration of data from the isogenic cell line screens enabled us to distinguish genes that are broadly essential in PC9 cells in erlotinib from gene knockouts that are negatively and positively selected in cells whose survival is driven by EGFR T790M/L858R (Fig. 3c), KRAS ${ }^{\text {G12V }}$ (Fig. 3d), or RIT1 ${ }^{\text {M90I }}$ (Fig. 3e), respectively. The landscape of these oncogene-specific dependencies differed in specific genes that recapitulated expected biology and pathway hierarchy. For example, in EGFR-mutant cells, top essential genes included known co-receptors and downstream pathway components ERBB3, SHC1, GRB2, PIK3CA, and PTPN11, also known as SHP2 (Fig. 3c, f, Supplementary Fig. 3a-d). In addition to these well-characterized EGFR downstream components, we found that erlotinib resistance conferred by $\mathrm{EGFR}^{\mathrm{T} 790 \mathrm{M} / \mathrm{L} 858 \mathrm{R}}$ was uniquely dependent on integrin-linked kinase $(I L K)$ and its binding partner LIMS1/PINCH1 (Fig. $3 \mathrm{~g}, \mathrm{~h}$ ). In cancer, ILK and adaptor proteins regulate interactions between tumor cells and the extracellular environment to activate signaling pathways that promote cell proliferation, migration, and epithelial-tomesenchymal transition ${ }^{37}$. In several human tumors, including non-small cell lung cancer, high ILK and LIMS1 expression 


\begin{abstract}
Fig. 3 Genome-wide identification of EGFR ${ }^{\text {T790M/L858R, KRAS }}{ }^{\text {G12V }}$, and RIT1 ${ }^{\text {M90I }}$ oncogene-specific genetic dependencies. a Venn Diagram showing the number of significant essential genes (CRISPR Score $<0.5$ and $p<0.05$ ) shared or specific to each isogenic PC9 cell line in erlotinib. $\mathbf{b}$ MSigDB overlap analysis of the enriched KEGG gene sets in the shared 813 genes from (a). c-e, Rank plots of CRISPR scores (CS) of erlotinib-treated vs. starting plasmid in (c), PC9-Cas9-EGFRT790M/L858R (d), PC9-Cas9-KRASG12V, and (e), PC9-Cas9-RIT1M90I. Key dependencies discussed in the text are labeled. Gray dashed lines mark genes with $|\mathrm{CS}|>0.5$. f-n Box plot showing the sgRNA abundance ( $\log _{2}$ reads per million) of sgRNAs targeting each indicated gene. Abundance in the plasmid library, early time point (ETP), or after 12 population doublings in vehicle (DMSO) or erlotinib was determined by PCR and Illumina sequencing. Box plots show the median (center line), first and third quartiles (box edges), and the min and max range (whiskers) of replicates. f-h sgRNAs

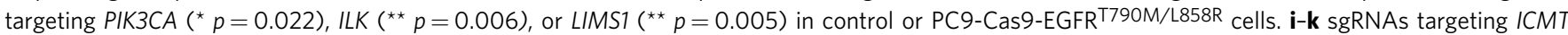
$\left(^{\star} p=0.025\right), C A M K 2 G\left({ }^{\star} p=0.045\right)$, or PDE11A ( $\left.{ }^{\star \star} p=0.004\right)$ in control or PC9-Cas9-KRASG12V. I-n sgRNAs targeting IGF1R $\left({ }^{\star} p=0.023\right)$, FURIN ( $\left.{ }^{\star \star} p=0.003\right)$, or CPD ( $\left.{ }^{\star \star} p=0.009\right)$ in control or PC9-Cas9-RIT1M90I cells. ${ }^{\star} p<0.05,{ }^{\star \star} p<0.01$, calculated by one-sided permutation testing using MAGeCK. For control PC9 cells ETP and DMSO, $n=3$ biological replicates. For oncogene-expressing PC9 cells DMSO and erlotinib, $n=2$ biological replicates. Source data are provided as a Source Data file.
\end{abstract}

correlates with increased disease progression ${ }^{38,39}$ and in EGFRmutant patients, correlates with significantly worse progressionfree survival after treatment with EGFR inhibitors ${ }^{40}$. Consistent with a role for ILK in mediating EGFR tyrosine kinase inhibitor (TKI) resistance, xenograft models of EGFR inhibitor-resistant human hepatocellular carcinoma cell lines found that inhibiting ILK activity increased the sensitivity of cells to EGFR inhibition ${ }^{41}$. Therefore, targeting ILK activity might be a valuable strategy for overcoming EGFR-TKI resistance in patients with $\mathrm{EGFR}^{\mathrm{T} 790 \mathrm{M} /}$ L858R mutations.

As predicted, KRAS-mutant cells were not reliant on upstream RTK signaling genes such as ERBB3, SHC1, or GRB2 for survival in erlotinib (Supplementary Fig. 3a-c). Although the tyrosine phosphatase gene PTPN11 has been recently identified as a potential therapeutic target in KRAS-mutant cancers ${ }^{42}$, PTPN11 knockout did not significantly impact $\mathrm{KRAS}^{\mathrm{G} 12 \mathrm{~V}}$-driven cell survival (Fig. 3d, Supplementary Fig. 3d). While this difference may be attributed to the different assay systems used, our finding is consistent with earlier reports showing that PTPN11 inhibition is lethal to cells with RTK activation but not to cells with oncogenic RAS proteins ${ }^{43}$. RIT1-mutant cells retained sensitivity to PTPN11 depletion (Fig. 3e, Supplementary Fig. 3d), highlighting a functional divergence between RIT1 ${ }^{\mathrm{M} 90 \mathrm{I}}$ and $\mathrm{KRAS}^{\mathrm{G} 12 \mathrm{~V}}$. Instead, KRAS ${ }^{\mathrm{G} 12 \mathrm{~V}}$-dependencies observed included ICMT (Fig. 3i), a methyltransferase responsible for the last step in a series of post-translational CAAX-domain modifications required for RAS to associate with the membrane ${ }^{44}$ and recently reported as a KRAS dependency ${ }^{20}$. RIT1 lacks a CAAX-domain and as predicted, RIT1-mutant cells did not rely on ICMT for survival in erlotinib (Fig. 3e). We observed enhanced sensitivity of KRAS ${ }^{\mathrm{G} 12 \mathrm{~V}}$ cells to the previously reported KRAS dependency, $X P O 1^{45}$. However, loss of XPO1 was lethal to all cell lines screened (Supplementary Fig. 3e). Additionally, we identified putative KRAS dependencies including CAMK2G, PDE11A, and SETD1A (Fig. 3j, k, Supplementary Fig. 3f).

Unlike KRAS-mutant cells, RIT1 ${ }^{\mathrm{M} 90 \mathrm{I}}$-mutant cells were sensitive to knockout of RTK and adaptor protein genes including PTPN11, GRB2, and EGFR itself (Fig. 3e, Supplementary Fig. 3c, d). Moreover, RIT1 ${ }^{\mathrm{M} 90 \mathrm{I}}$-mutant cells required insulin-like growth factor receptor 1 (IGF1R) (Fig. 3l) and several related components of IGF1R signaling. IGF1R is a multifunctional receptor that promotes cell proliferation, differentiation, and survival through activation of the PI3K-AKT and RAS/MAPK signaling pathways ${ }^{46}$. IGF1R is synthesized as an inactive precursor proprotein, which requires endoproteolytic cleavage to gain full biological activity 47,48 . In addition to IGF1R, RIT1-mutant cells were dependent on the proprotein convertase FURIN (Fig. $3 \mathrm{~m}$ ), and carboxypeptidase D (Fig. 3n), two proteins directly involved in IGF1R maturation and activity 49,50 .

To validate the genetic dependencies identified and determine the robustness of these screening results, we designed a custom
sgRNA library consisting of 1000 non-targeting sgRNAs and 10,333 unique sgRNA sequences targeting 1288 genes (Methods, Supplementary Data 5). We performed secondary screening in three replicates each of vehicle- and erlotinib-treated PC9-Cas9RIT1 ${ }^{\text {M90I }}$ cells. Results from the primary screen and validation screen were highly correlated $\left(R^{2}=0.77\right.$; Supplementary Fig. $\left.3 \mathrm{~g}\right)$, with $75.4 \%$ of essential genes validating and $100 \%$ of positively selected genes validating in the secondary screen.

Together, these data illustrate the utility of CRISPR screens in isogenic cell lines for the identification of oncogene dependencies in cancer. Comparative analysis of EGFR ${ }^{\mathrm{T} 790 M / L 858 R}$, KRASG12V, and RIT1 ${ }^{\text {M90I }}$ dependencies confirmed expectations from established pathway hierarchy. Moreover, the high validation rate in the secondary screen demonstrates the reproducibility of the majority of identified dependencies.

Enhanced sensitivity of RIT1-mutant cells to loss of mitotic regulators. Next, we sought to further investigate the different dependencies conferred by mutant RIT1 and KRAS. RIT1-mutant cells were dependent on several known positive regulators of RAS signaling, including SOS1 and SHOC2 (Fig. 4a), while loss of negative regulators of RAS and genes commonly mutated in Noonan syndrome, such as NF1, SPRED1, and LZTR1, promoted RIT1-induced cell proliferation in erlotinib (Fig. 4a). In addition to regulators of RAS signaling, several RIT1 dependencies were involved in mitotic spindle assembly and cell cycle regulation (Fig. 4a, b). A top RIT1-mutant dependency was USP9X, which encodes a deubiquitinase with many target substrates ${ }^{51-53}$ (Fig. 3e, Fig. 4a). During mitosis, USP9X plays an important role in regulating anaphase initiation and chromosome segregation by stabilizing key mitotic regulators such as Survivin and CDC20 $21-54$. Knockout of USP9X was significantly depleted in

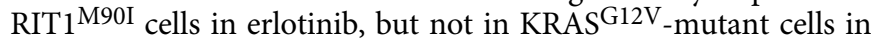
erlotinib or control PC9 cells in DMSO (Fig. 4a). In addition to USP9X, RIT1-mutant cells were sensitive to depletion of mitotic regulators including $A U R K A, A U R K B$, and $M A D 2 L 1 B P / p 31^{\text {comet }}$ (Fig. 4a). Pathway enrichment analysis confirmed that cell cycle and mitotic regulators were significantly depleted in RIT1-mutant cells (Fig. 4b). This effect could conceivably be due to differences in proliferation rates; however, doubling rates were identical between the two cell lines (Fig. 2c). Secondary screening confirmed the enhanced sensitivity of RIT1-mutant cells to depletion of USP9X and AURKA (Supplementary Fig. 3g, Supplementary Fig. 4a, b) along with the RAS pathway and Noonan syndrome genes SHOC2 and SOS1 (Supplementary Fig. 3g, Supplementary Fig. $4 \mathrm{a}, \mathrm{b})$. To further validate the RIT1-specific dependency on USP9X, SHOC2 and AURKA, we individually knocked out each gene in PC9-Cas9-RIT1 ${ }^{\text {M90I }}$ cells (Supplementary Fig. 4c, Methods, Supplementary Table 1). Consistent with the pooled CRISPR screen results, individual loss of SHOC2, AURKA, or 
a

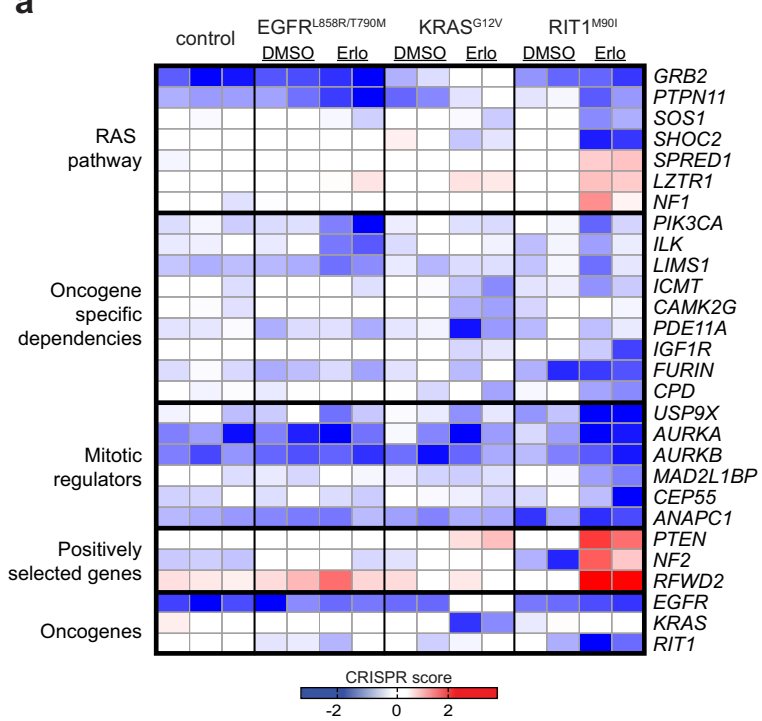

C

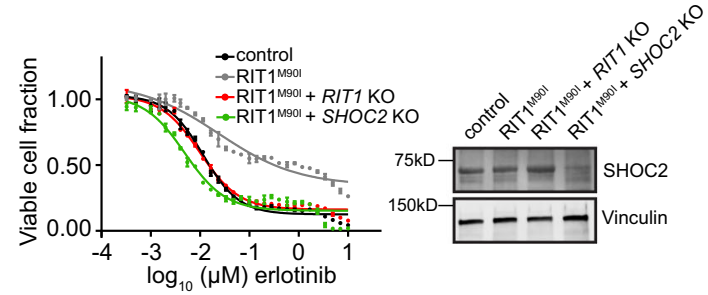

d

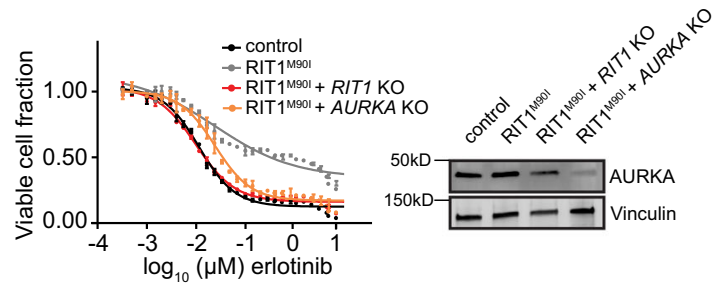

e

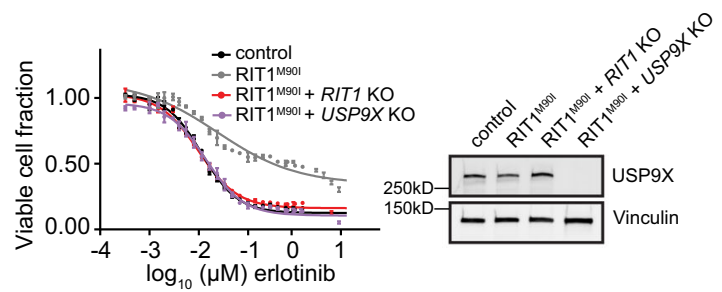

f

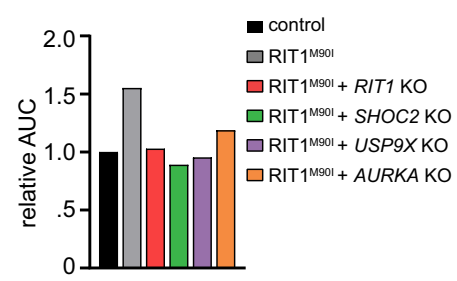

USP9X each resulted in restored sensitivity to erlotinib (Fig. 4c-f) and osimertinib (Supplementary Fig. 4d, e).

To determine whether these oncogenic RIT1 dependencies extended to other cell contexts and in the absence of erlotinib treatment, we evaluated their function in NCI-H2110, a

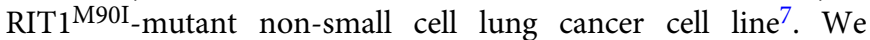
introduced a doxycycline-regulated Cas9 (iCas9) construct ${ }^{55}$ into b

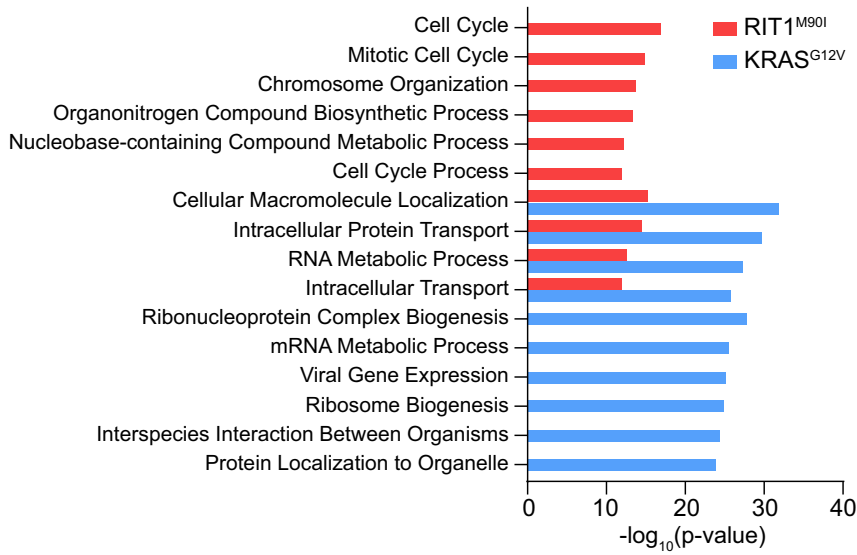

g
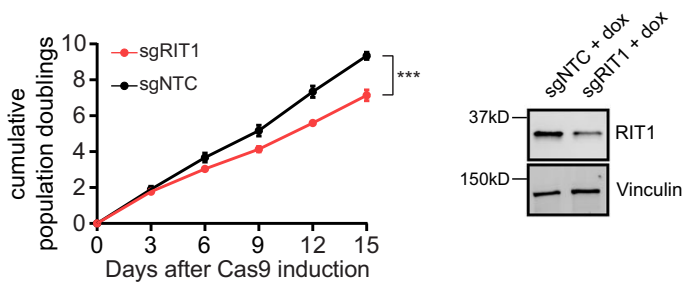

h
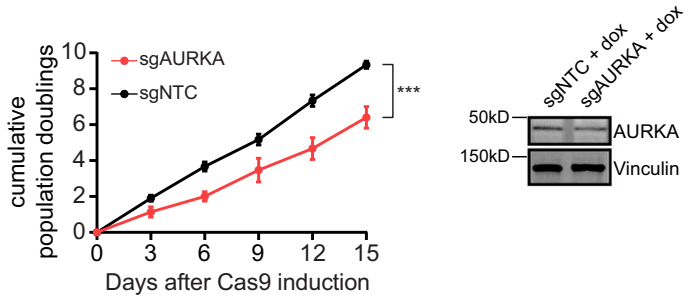

i
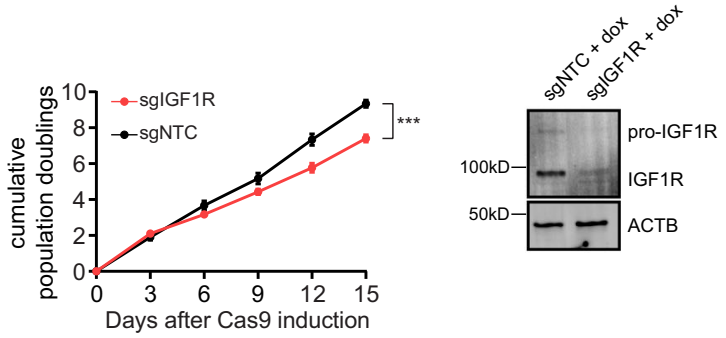

j
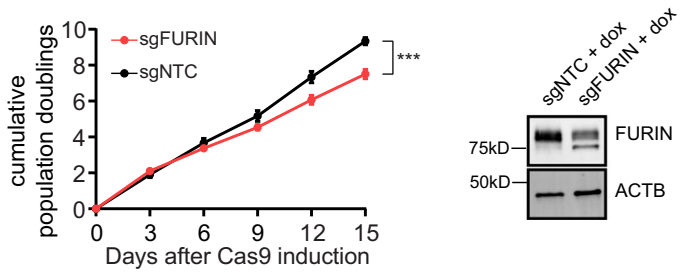

NCI-H2110 cells, and then introduced individual RIT1, AURKA, IGF1R, and FURIN sgRNAs into pools of H2110iCas9 cells (Methods, Supplementary Table 2). Sanger sequencing and ICE analysis $^{56}$ after five days of Cas9 induction found that $28-52 \%$ of each cell pool contained an indel mutation (Supplementary Fig. 4f), which in all cases resulted in decreased protein expression (Fig. 4g-j). Supporting a requirement for RIT1, 
Fig. 4 RIT1-mutant cells are vulnerable to loss of RAS pathway and cell cycle genes. a Heatmap illustrating the CS of selected dependencies clustered by biological pathway. Each column is a different replicate and shows CS between erlotinib or DMSO versus plasmid. b MSigDB overlap analysis of the GO Biological Process gene sets significantly enriched in the top 500 PC9-Cas9-RIT1M90I and PC9-Cas9-KRASG12V essential genes. c-e Left, 96-hour dose-response curve of erlotinib in clonal SHOC2 knockout (KO), AURKA KO, or USP9X KO cells derived from PC9-Cas9-RIT1M90I cells. The same data for control and PC9-Cas9-RIT1 ${ }^{\mathrm{M} 90 \mathrm{I}}$ and RIT1 KO cells are plotted on each panel for reference. Data shown are the mean \pm s.d. of two technical replicates. Right, Western blot for SHOC2, AURKA, or USP9X expression in clonal knockout cells derived from PC9-Cas9-RIT1M90I cells. Vinculin was used as a loading control. f Area-under-the-curve (AUC) analysis of data from panels (c-e). $\mathbf{g - j}$ - Left, Cumulative population doublings of H2110iCas9 cells grown in the presence of doxycycline ( + dox) expressing the indicated sgRNAs compared to cells expressing non-targeting control sgRNA (sgNTC). The same data for sgNTC is shown on each panel for reference. Data shown are the mean \pm s.d. of three technical replicates. ${ }^{\star \star \star} p<0.001\left(\mathbf{g}^{\star \star \star} p=0.0005 . \mathbf{h}{ }^{\star \star \star} p=0.001\right.$. $\mathbf{i}^{\star \star \star} p=0.0003 . \mathbf{j}^{\star \star \star} p=0.0007$.) by unpaired two-tailed t-test. Right, Western blot of day 5 lysates generated from parental H2110iCas 9 or pooled $R I T 1$ KO, AURKA KO, IGFIR KO, or FURIN KO cells. Vinculin or Actin (ACTB) was used as a loading control. Source data are provided as a Source Data file.

a

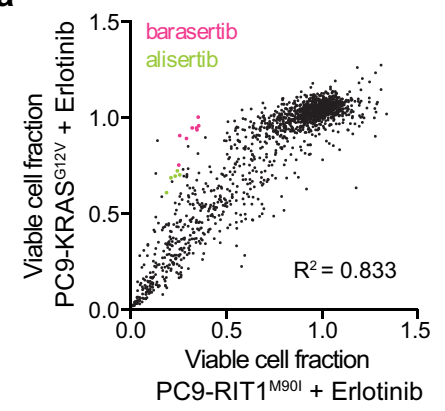

b

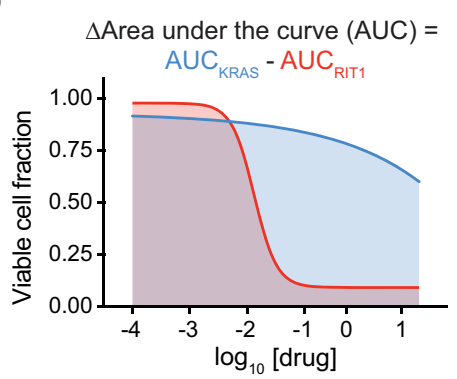

C

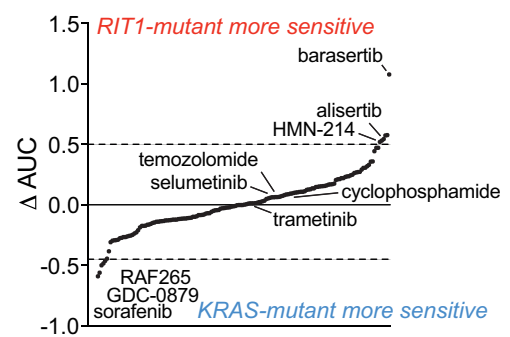

d
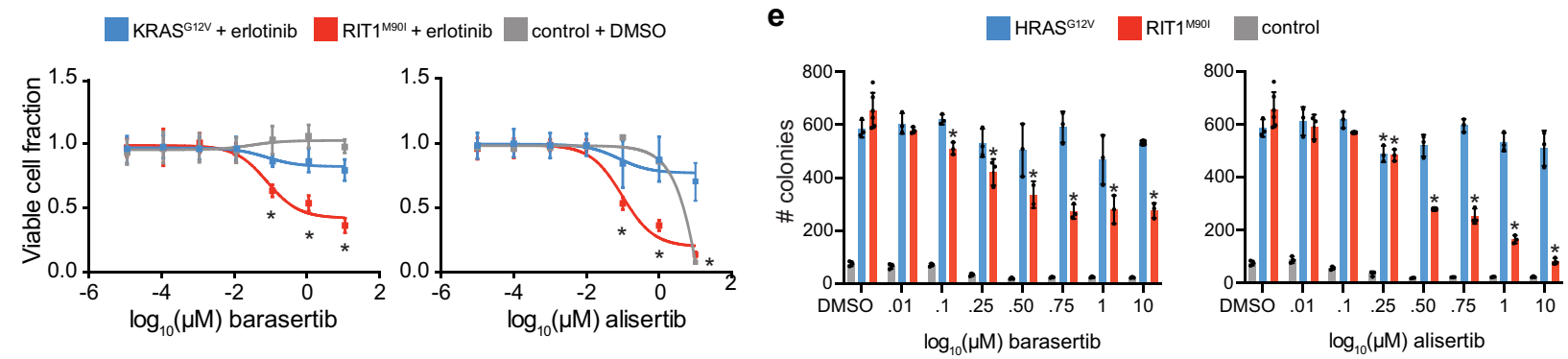

Fig. 5 RIT1-mutant cells are sensitive to Aurora kinase inhibition. a Drug sensitivity screen of 160 small molecules, 8 doses per compound, in isogenic PC9-RIT1 ${ }^{\mathrm{M} 90 \mathrm{I}}$ and PC9-KRASG12V treated in combination with each test condition and $500 \mathrm{nM}$ erlotinib for 96-hours. Data shown are the mean of two replicates of each individual dose/compound. Viable cell fraction was calculated by normalizing CellTiterGlo luminescence in the test condition to CellTiterGlo luminescence of cells treated only with $500 \mathrm{nM}$ erlotinib. b Schematic illustrating differential 'area-under-the-curve' (AUC) analysis. c $\Delta$ AUC analysis of 160 small molecules generated from data shown in (a) and calculated as in (b). Each dot represents a different compound. $\triangle A U C$ are mediancentered and plotted from least to greatest. $\mathbf{d}$ Validation of enhanced response to barasertib, left, or alisertib, right. Data shown are mean $\pm \mathrm{s}$.d. of $\mathrm{n}=16$ replicates per dose. ${ }^{*} p<0.05$ by unpaired two-tailed t-test. e Soft agar colony formation in NIH3T3 cells expressing RIT1 ${ }^{\mathrm{M} 90 \mathrm{I}}$, HRASG12V or empty vector (control). Data shown are the mean \pm s.d. of $n=3+$ replicates for all conditions except for the $0.5 \mu \mathrm{M}$ barasertib-treated RIT1M90I cells $(n=2)$. ${ }^{*} p<0.05$ by unpaired two-tailed t-test. Source data are provided as a Source Data file.

AURKA, IGF1R, and FURIN in the proliferation of RIT1-mutant lung cancer, knockout of each gene significantly reduced cell proliferation of H2110iCas9 compared to cells expressing a nontargeting control guide (sgNTC) (Fig. 4g-j). These data suggest that RIT1 candidate dependencies identified through pooled genome-wide CRISPR screening may represent true dependencies in naturally occurring RIT1-mutant lung adenocarcinomas and point to a specific vulnerability of RIT1 ${ }^{\mathrm{M} 90 \mathrm{I}}$-mutant cells to loss of mitotic regulators, particularly those involved in the spindle assembly checkpoint.

RIT1-mutant cells are sensitive to Aurora kinase inhibition. To further explore unique therapeutic vulnerabilities of RIT1-mutant cells, we used the same PC9 isogenic system to evaluate therapeutic efficacy of 160 small molecules, the majority of which are in clinical use or development. PC9-RIT1 ${ }^{\text {M90I }}$ and PC9$\mathrm{KRAS}^{\mathrm{G} 12 \mathrm{~V}}$ were co-treated with erlotinib and each of the compounds at varying doses (eight per compound). The majority of compounds affected PC9-RIT1 ${ }^{\text {M90I }}$ and PC9-KRAS ${ }^{\text {G12V }}$ cells with similar efficacy $\left(R^{2}=0.83\right.$; Fig. 5a, Supplementary Data 6$)$. Comparison of the area under the curve (AUC) for each compound's dose-response in each cell line (Fig. 5b) revealed that RIT1- and KRAS-mutant cells shared sensitivity to MEK inhibitors selumetinib and trametinib while sharing resistance to alkylating agents such as temozolomide and cyclophosphamide (Fig. 5c, Supplementary Data 6). A few compounds showed modest selectivity for KRAS-mutant cells, including three of the seven RAF inhibitors in the screen: sorafenib, RAF265, and GDC0879, whereas top differentially sensitive compounds in PC9RIT1 $^{\text {M90I }}$ cells were the Aurora kinase inhibitors alisertib, an inhibitor of Aurora A, and barasertib, an inhibitor of Aurora B (Fig. 5c). A PLK1 inhibitor, HMN-214 was also more effective in PC9-RIT1 ${ }^{\text {M90I }}$ cells. Interestingly, Aurora A, Aurora B, and PLK1 are all important mitotic regulators with multifaceted functions including regulation of the spindle assembly checkpoint ${ }^{57}$. 
We validated these findings not only in repeated PC9 drug response assays (Fig. 5d), but also in an independent cellular context, RIT1-mediated cell transformation in NIH3T3 cells. We found that both alisertib and barasertib treatment suppressed RIT1 ${ }^{\text {M90I }}$-driven anchorage-independent growth, whereas transformation by oncogenic RAS was largely unaffected by Aurora inhibition (Fig. 5e). Together, these drug response and CRISPR genetic assays point to a unique sensitivity of RIT1-mutant cells to perturbation of mitotic regulators. Although genes such as Aurora kinases are generally essential in most cells ${ }^{58}$, our data suggest that RIT1-mutant cells may be unusually vulnerable to inhibition of these factors, which prompted us to investigate the mechanistic basis of this sensitivity.

RIT1 ${ }^{\text {M90I }}$ weakens the spindle assembly checkpoint. Fidelity of mitosis relies on the spindle assembly checkpoint (SAC), a critical cellular pathway that senses unaligned kinetochores and arrests mitotic progression during metaphase by inhibiting the anaphasepromoting complex/cyclosome (APC/C) until kinetochores are properly attached to microtubules ${ }^{57}$. Altering the SAC in normal cells accelerates mitotic timing ${ }^{59}$, and in conditions of mitotic stress can either promote mitotic cell death or result in mitotic slippage, the exit from mitosis before proper chromosome alignment is complete ${ }^{60}$. Given that many of the RIT1 dependencies identified were components of the SAC (e.g. USP9X, Aurora kinases, MAD2L1BP), we hypothesized that RIT1 ${ }^{\text {M90I }}$ might weaken the SAC, enhancing the vulnerability of the cells to further loss of SAC activity. To test this hypothesis, we adapted a model system commonly used for mitotic timing experiments ${ }^{61}$, HeLa cells expressing a nuclear H2B-GFP fusion protein ${ }^{62}$ (Fig. 6a). We used live-cell fluorescence microscopy to time the duration of mitosis from nuclear envelope breakdown (NEBD) to anaphase onset ${ }^{59}$ (Fig. 6b). In parental H2B-GFP cells the median duration of mitosis was $70.5 \mathrm{~min}(95 \% \mathrm{CI}=63-82 \mathrm{~min})$, while in

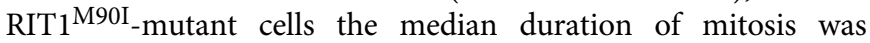
reduced to $48 \mathrm{~min}$ ( $95 \% \mathrm{CI}=45-51 \mathrm{~min}$ ) (Fig. 6c, d). Overall mitotic index was unaffected, suggesting that mitotic entry is not regulated by $\mathrm{RIT}^{\mathrm{M} 90 \mathrm{I}}$ (Fig. 6e). The difference in mitotic timing between RIT1 ${ }^{\mathrm{M} 90 \mathrm{I}}$-mutant cells and parental cells was eliminated by treatment with reversine, an inhibitor of the MPS1 kinase involved in establishing the SAC kinetochore signal, demonstrating that RIT1 ${ }^{\mathrm{M} 90 \mathrm{I}}$ perturbs mitotic timing at the level of the SAC (Fig. 6c, d). A weakened SAC via perturbation of key components has been associated with mitotic errors including misaligned chromosomes, chromosome bridges, micronuclei formation, and aneuploidy ${ }^{63-66}$. Consistent with RIT1 ${ }^{\text {M90I }}$ suppression of the SAC, RIT1-mutant cells showed significantly higher prevalence of chromosomal abnormalities compared to parental cells (Fig. 6f, g).

The vulnerability of RIT1-mutant cells to Aurora A inhibition and RIT1's ability to weaken the SAC led us to hypothesize that Aurora A inhibition may be able to overcome the mitotic phenotype induced by $\mathrm{RIT} 1^{\mathrm{M} 90 \mathrm{I}}$. To test this hypothesis, we performed mitotic timing analysis in HeLa H2B-GFP cells treated with the Aurora A inhibitor alisertib. Whereas RIT1 ${ }^{\mathrm{M} 90 \mathrm{I}}$ alone accelerated mitosis compared to parental cells, mitotic timing in RIT1 ${ }^{\text {M90I }}$ cells was increased compared to parental in the setting of alisertib (Fig. 6h). RIT1 ${ }^{\mathrm{M} 90 \mathrm{I}}$-mutant cells accumulated more mitotic errors than parental cells in alisertib (Fig. 6f). Taken together, these data indicate that oncogenic RIT1 weakens the SAC, creating a vulnerability to Aurora kinase inhibitors. Because Aurora kinases are required for full activation of the $S A C^{53,67,68}$, we propose a working model by which combined RIT1 ${ }^{\mathrm{M} 90 \mathrm{I}}$ and Aurora A inhibition leads to cellular toxicity and cell death (Fig. 6i).
YAP1 activation synergizes with RIT1 ${ }^{\text {M90I }}$ to promote lung cancer. In addition to differences in genetic dependencies, each oncogene displayed substantial differences in the landscape of positively selected gene knockouts (Fig. 7a-c). PTEN knockout, which is known to promote erlotinib resistance ${ }^{69,70}$, cooperated with both KRASG12V and RIT1 ${ }^{\text {M90I }}$ but showed no evidence of selection in EGFR $^{\text {T790M/L858R }}$ cells (Supplementary Fig. 5a), consistent with EGFR's ability to activate the PI3K pathway on its own. In contrast, drug resistance driven by $\mathrm{EGFR}^{\mathrm{T} 790 \mathrm{M} / \mathrm{L} 858 \mathrm{R}}$ was promoted by knockout of CSK (Fig. 7a), which encodes C-terminal SRC kinase, a negative regulator of $\mathrm{SRC}^{71}$. SRC activates EGFR via phosphorylation, to enhance EGFR kinase activity and prolong signaling by suppressing EGFR degradation ${ }^{72}$. Enrichment for CSK knockout was not observed in KRAS- or RIT1-mutant PC9 cells (Fig. 7b, c). Whereas EGFR-mutant and KRAS-mutant cells showed few cooperating events ( $n=37$ and $n=5$ knockouts, respectively), RIT1 ${ }^{\text {M90I }}$ mutant cells had 152 significantly enriched gene knockouts in erlotinib (Fig. 7c). Five of the top 12 genes whose loss synergized with RIT1 ${ }^{\text {M901 }}$ belong to the Hippo pathway: NF2, CAB39, WWC1, TAOK2, and SAV1 (Supplementary Fig. 5b-f). Among all positively selected gene knockouts $(p<0.05, \mathrm{CS}>0.5)$, pathway enrichment analysis using the Molecular Signatures Database (MSigDB) identified the Hippo signaling pathway as the top REACTOME pathway $($ FDR $=2.08 \mathrm{e}-5)$ (Fig. 7d, e). Secondary screen validation confirmed robust enrichment of Hippo pathway knockouts including NF2, CAB39, and TAOK1 in RIT1 ${ }^{\mathrm{M} 90 \mathrm{I}}$-mutant cells (Supplementary Fig. 3g, Supplementary Fig. 5g). Validation experiments using pooled and clonal knockout cells demonstrated that loss of NF2 cooperated with $\mathrm{RIT} 1^{\mathrm{M} 90 \mathrm{I}}$ to promote erlotinib resistance (Supplementary Fig. 5h, i).

The Hippo pathway is a tumor-suppressive cellular signaling pathway that regulates proliferation and cell survival via negative regulation of YAP1 stability and nuclear translocation ${ }^{73}$. Enrichment for loss of Hippo pathway genes in RIT1 ${ }^{\mathrm{M} 90 \mathrm{I}}$ cells suggests that inactivation of Hippo signaling may synergize with oncogenic RIT1 to promote cellular proliferation and survival. However, YAP1 activation has previously been shown to promote erlotinib resistance itself ${ }^{74}$, so we tested whether YAP1 activation synergizes with $\mathrm{RIT1}^{\mathrm{M} 90 \mathrm{I}}$ in the absence of erlotinib using a human small airway lung epithelial (SALE) cell transformation model $^{8}$. To test whether RIT1 ${ }^{\text {M90I }}$ or activated YAP1 can transform these cells, we expressed RIT1 ${ }^{\mathrm{M} 90 \mathrm{I}}$ alone or in combination with YAP1 $1^{5 \mathrm{SA}}$, which harbors five serine-toalanine mutations at critical LATS1/2 phosphorylation sites, resulting in a stabilized, nuclear-localized YAP1 protein ${ }^{75}$. The co-expression of RIT1 $1^{\mathrm{M} 90 \mathrm{I}}$ and YAP $1^{5 \mathrm{SA}}$ in SALE cells caused the cells to shift from an adherent to a suspension growth phenotype (Fig. 7f, Supplementary Fig. 5j). Moreover, co-expression of RIT1 ${ }^{\mathrm{M} 90 \mathrm{I}}$ and YAP $1^{5 \mathrm{SA}}$ synergistically transformed SALE cells to induce xenograft tumor formation (Fig. 7g, Supplementary Fig. 5j). Similar results were obtained by knocking out NF2 in combination with RIT1 expression in SALE cells (Fig. 7h, Supplementary Fig. 5k). Together these data demonstrate that oncogenic RIT1 and activated YAP1 cooperate to promote tumorigenesis.

Next, we leveraged the finding that RIT1 ${ }^{\text {M90I }}$ and YAP15SA synergize to promote tumorigenesis in SALE cells to expand the validation of oncogenic RIT1 dependencies AURKA, IGF1R, and FURIN in vivo. We introduced doxycycline-inducible Cas9 into SALE cells co-expressing RIT1 ${ }^{\mathrm{M} 90 \mathrm{I}}$ and YAP1 ${ }^{5 \mathrm{SA}}\left(\mathrm{RIT}^{\mathrm{M} 90 \mathrm{I}}+\right.$ YAP1 ${ }^{5 S A}$ ) followed by transduction with sgNTC, sgRIT1, sgAURKA, sgIGF1R, or sgFURIN constructs. Doxycyclinetreatment of xenografts of $\mathrm{RIT} 1^{\mathrm{M} 90 \mathrm{I}}+\mathrm{YAP} 1^{5 \mathrm{SA}}$ xenografts resulted in significant inhibition of tumor growth compared to sgNTC control (Fig. 7i-l, Supplementary Fig. 51-o). These data extend the cellular contexts in which these RIT1 dependencies are 


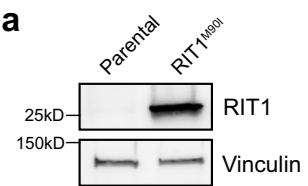

b

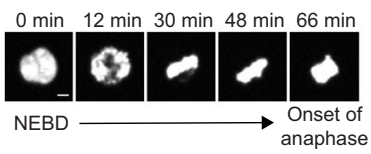

f

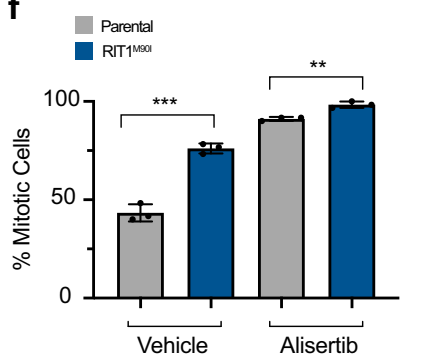

C

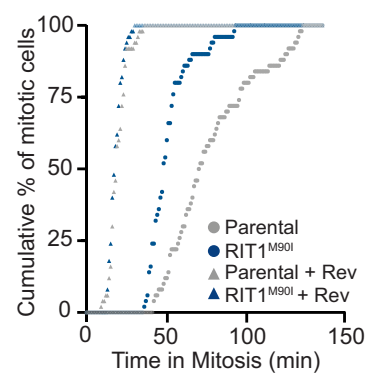

d

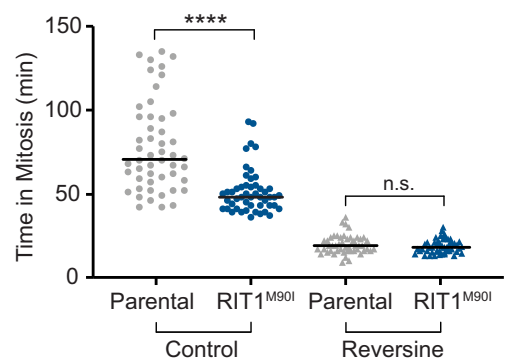

e

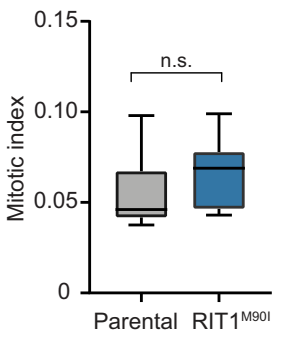

g

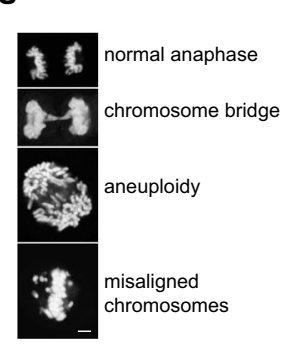

h

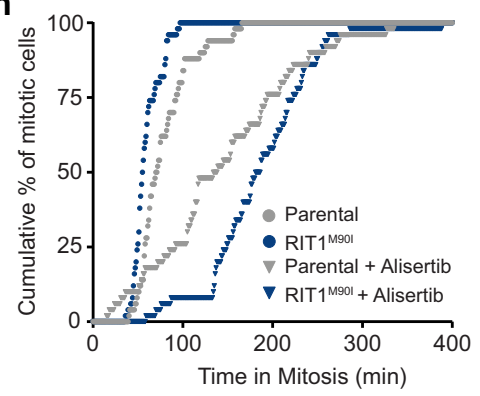

i

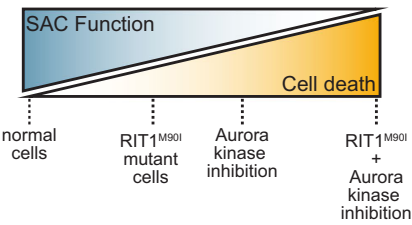

Fig. 6 RIT1M90I weakens the spindle assembly checkpoint. a Western blot of RIT1 expression in parental and RIT1M90I-expressing HeLa H2B-GFP cells. Vinculin was used as a loading control. $\mathbf{b}$ Duration of mitosis was measured as time from nuclear envelope breakdown (NEBD) to the onset of anaphase. Each frame represents movie stills from time-lapse live-cell imaging of parental HeLa H2B-GFP cells undergoing mitosis, scale bar $=5 \mu \mathrm{m}$. c Time-lapse fluorescence microscopy of time in mitosis in asynchronous parental and RIT1 ${ }^{\mathrm{M} 90 \mathrm{O}}$-expressing HeLa H2B-GFP cells in normal media conditions (control) or treated with 0.5 $\mu \mathrm{M}$ reversine (Rev) two hours before imaging. $n=50$ cells per condition. Mitotic timing was measured from time of NEBD to anaphase onset. $\mathbf{d}$ Alternative representation of data from (c). Data shown are the mean and individual data points of each condition. ${ }^{\star \star \star \star} p=9.06 \mathrm{e}^{-9}$, n.s., $p=0.1754$ by unpaired two-tailed ttest. e Mitotic index calculated as the percentage of mitotic cells in a frame at a chosen time point. Box plots show the median (center line), first and third quartiles (box edges), and the min and max range (whiskers). $n=2$ biological replicates, 6 time points per condition, n.s., $p=0.3587$ by unpaired two-tailed ttest. $\mathbf{f}$ Comparison of mitotic error rates in HeLa H2B-GFP cells expressing RIT1M90I compared to parental HeLa H2B-GFP cells in vehicle (DMSO)-treated and alisertib-treated $(1 \mu \mathrm{M})$ conditions. $n=3$ biological replicates, error bars indicate s.d., ${ }^{\star \star \star} p=0.0004,{ }^{\star \star} p=0.0029$ by unpaired two-tailed t-test. $\mathbf{g}$ Representative images of mitotic errors from HeLa cells expressing RIT1M9OI and quantified in (f), scale bar $=5 \mu \mathrm{m}$. $\mathbf{h}$ Time-lapse fluorescence microscopy of time in mitosis in asynchronous parental and RIT1 ${ }^{\mathrm{M} 90 \mathrm{I}}$-expressing HeLa H2B-GFP cells in normal media conditions or treated with $1 \mu \mathrm{M}$ alisertib two hours before imaging. $n=50$ cells per condition. i Proposed model explaining enhanced efficacy of Aurora kinase inhibitors in RIT1 ${ }^{\mathrm{M} 90 \mathrm{I}}$-mutant cells. Source data are provided as a Source Data file.

found to be critical for RIT1 function and confirm that these genes are relevant not only for RIT1-driven drug resistance but also for tumorigenesis.

YAP1 activation and downregulation of Hippo pathway genes in human RIT1-mutant lung cancer. To investigate whether coactivation of YAP1 and RIT1 occurs in human lung cancer, we analyzed data from 230 human lung adenocarcinomas sequenced by The Cancer Genome Atlas ${ }^{6}$. Because wild-type RIT1 overexpression can transform cells ${ }^{8}$ and may confer Noonan syndrome in individuals with germline LZTR1 mutations ${ }^{15}$, we included RIT1-amplified tumors in our analysis. 16\% of lung adenocarcinomas harbored mutated or amplified RIT1 (Supplementary Fig. 6a). Tumors showed copy number-related increases in RIT1 mRNA expression, indicating that RIT1-amplified tumors overexpress RIT1 (Supplementary Fig. 6b). Analysis of differentially expressed genes in RIT1-mutant and RIT1-amplified lung adenocarcinomas identified significant loss of expression of Hippo pathway genes $(\mathrm{FDR}=6 \mathrm{e}-4)$. Driving this enrichment was the downregulation of key Hippo pathway genes STK4, SAV1, TAOK3, MAP4K5, STK38L, and AMOTL1 (Fig. 8a). Other significantly altered pathways included EGFR inhibitor resistance $(\mathrm{FDR}=2.09 \mathrm{E}-6), \mathrm{PI} 3 \mathrm{~K}-\mathrm{AKT} \quad(\mathrm{FDR}=2.09 \mathrm{E}-6)$, and MAPK signaling $(\mathrm{FDR}=7.6 \mathrm{E}-4)$ (Supplementary Data 7$)$. In contrast, the WNT-pathway gene DVL2 was overexpressed (Fig. 8a), which may further activate YAP1 via crosstalk between WNT and Hippo signaling networks ${ }^{76,77}$. 76\% (28/37) of RIT1-altered tumors had low expression of at least one Hippo pathway gene, compared to $46 \%$ (88/193) of tumors with wild-type/normal RIT1 (Fig. $8 \mathrm{~b} ;{ }^{* * *} p<0.001$ by one-sided Fisher's exact test).

To confirm this observation in an independent clinical cohort, we identified RIT1-mutant non-small cell lung tumors using MSK-IMPACT sequencing ${ }^{78}$ (Supplementary Data 8). We performed YAP1 immunohistochemistry (IHC) comparing YAP1 localization in RIT1-mutant tumors to tumors with known YAP1 activation by chromosomal amplification of YAP1 or $Y_{E S 1}{ }^{79}$, or control tumors with wild-type RIT1 and YAP1/YES1 (Fig. 8c). When Hippo signaling is suppressed, YAP1 becomes unphosphorylated and translocates to the nucleus to regulate transcription $^{80}$. Indicative of YAP1 activation, RIT1-mutant tumors had significantly higher YAP1 nuclear $\mathrm{H}$-scores than control tumors, similar to that seen in YAP1-amplified tumors (Fig. 8c, d). Together, these data show that Hippo inactivation synergizes with mutant RIT1 in cancer models, and Hippo pathway inactivation and YAP1 activation also occurs in RIT1altered human lung tumors.

To investigate the molecular basis for the observed functional synergy, we performed RNA-seq in SALE cells expressing 
a

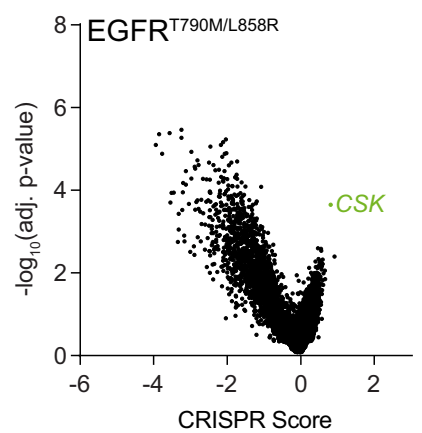

b

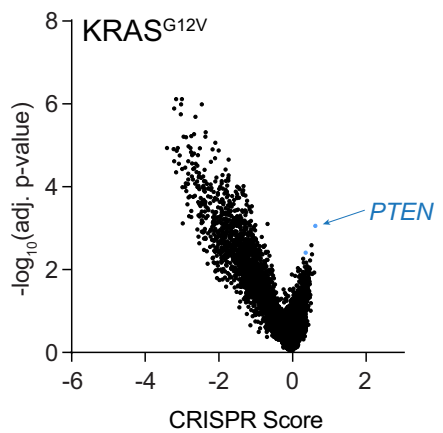

C

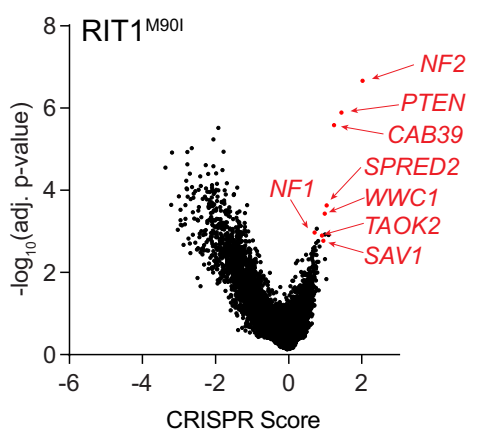

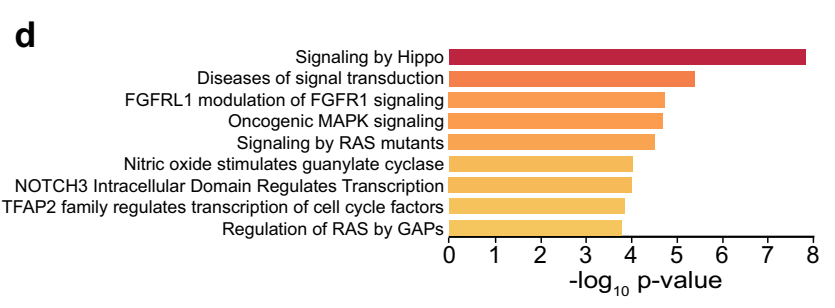

f

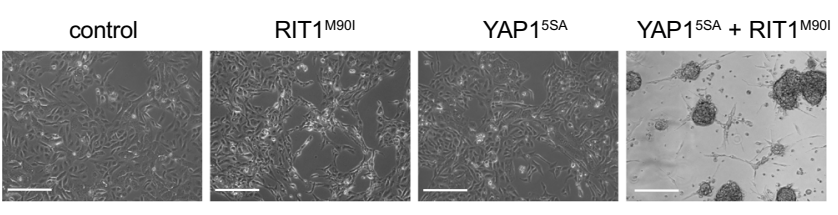

e

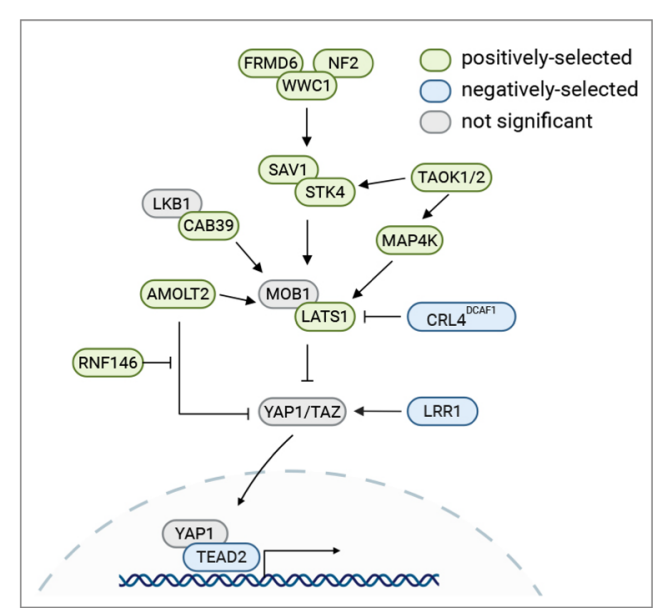

g

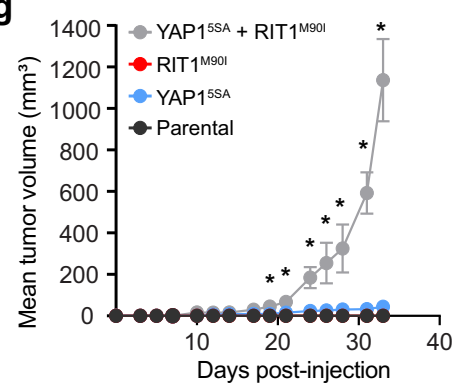

j

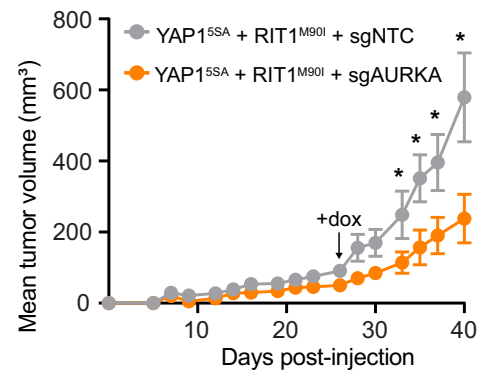

h

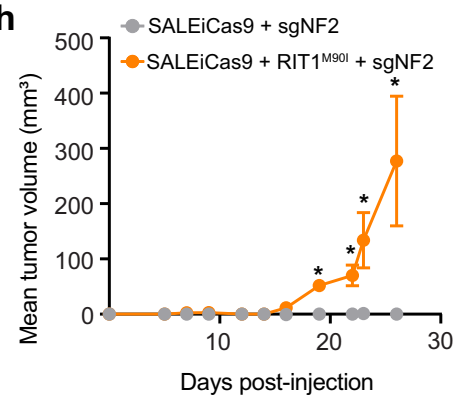

$\mathbf{k}$

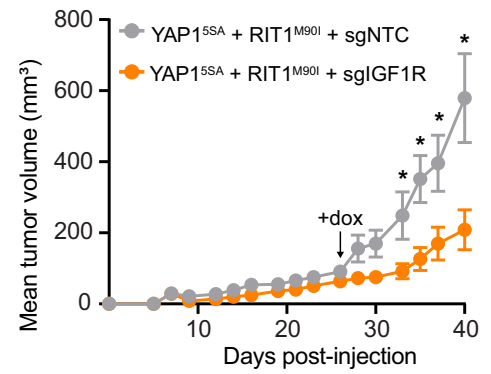

i

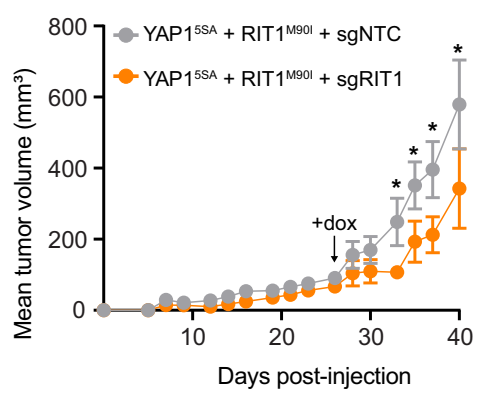

I

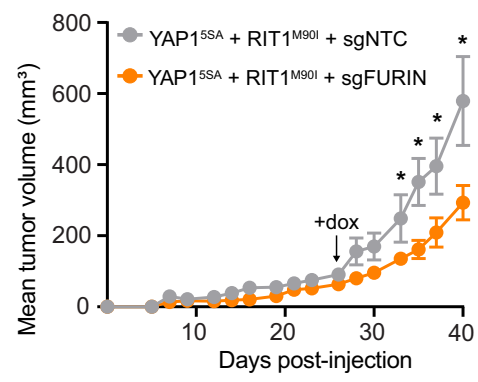

RIT1 ${ }^{\mathrm{M} 90 \mathrm{I}}$ and YAP $1^{5 \mathrm{SA}}$ individually or in combination (Supplementary Fig. 6c-e, Supplementary Data 9). Interestingly, $\mathrm{RIT1}^{\mathrm{M} 90 \mathrm{I}}$ and YAP1 ${ }^{5 \mathrm{SA}}$ induced very different transcriptional states with little correlation (Supplementary Fig. 6c, left panel). Transcripts altered by RIT1 ${ }^{\text {M90I }}$ were not enriched for a YAP1 gene signature ${ }^{81}$, whereas YAP1 ${ }^{5 S A}$ drove activation of this signature as expected (Supplementary Fig. 6d). However, when
RIT1 $^{\text {M90I }}$ and YAP1 ${ }^{5 S A}$ were co-expressed, YAP1-regulated gene expression was enhanced by RIT1 ${ }^{\mathrm{M} 90 \mathrm{I}}$ compared to YAP15SA alone (Supplementary Fig. 6c, e), with known YAP1 targets such as TNNT2, ITGB2, and COL4A3 showing 2.5-3.6 fold increased expression compared to YAP1 $1^{5 S A}$ alone (Supplementary Fig. 6e), which was confirmed by qRT-PCR (Supplementary Fig. 6f) Although the specific mechanism of RIT1 and YAP1 synergy 
Fig. 7 Hippo pathway inactivation synergizes with RIT1M90I to promote cell survival and proliferation. a-c Volcano plots of genome-wide CRISPR screening data from (a) PC9-Cas9-EGFR ${ }^{\text {T790M/L858R }}$ cells, (b) PC9-Cas9-KRASG12V cells and (c) PC9-Cas9-RIT1M90I cells cultured in 40 nM erlotinib for 12 population doublings. CRISPR Score indicates the normalized $\log _{2}$ (fold-change) of the average of 4 sgRNAs per gene in two biological replicates in erlotinib compared to the starting abundance in the plasmid library. d MSigDB overlap analysis of the top REACTOME gene sets in RIT1M90I positively selected gene knockouts $(p<0.05$, CRISPR score $>0.5$ ). e Depiction of positively selected (green) and negatively selected (blue) Hippo pathway components identified in PC9-Cas9-RIT1 ${ }^{\mathrm{M} 90 \mathrm{I}}(|\mathrm{CS}|>0.5$ and $p<0.05)$. Schematic created partially with Biorender.com. $\mathbf{f}$ Phase contrast images of SALEiCas9 cells (control) or expressing RIT1M90I, the constitutively nuclear-localized YAP15SA, or RIT1M90I and YAP15SA. Scale bar $=200 \mu$ m.

Representative images from $n=3$ independent experiments are shown. $\mathbf{g}$ Xenograft assay of SALEiCas9 cells expressing RIT1M90I, YAP15SA, or combined $\mathrm{RIT1}^{\mathrm{M} 90 \mathrm{I}}$ and YAP15SA in immunocompromised mice. Data shown are the mean \pm s.e.m. of $n=6$ tumors per group. ${ }^{*} p<0.05$ by unpaired two-tailed t-test. $\mathbf{h}$ Xenograft assay of SALEiCas9 cells expressing sgNF2, or combined RIT1M90I and sgNF2 in immunocompromised mice. Data shown are the mean \pm s.e.m. of $\mathrm{n}=8$ tumors per group. ${ }^{\star} p<0.05$ by unpaired two-tailed t-test. $\mathbf{i}-\mathbf{I}$ Xenograft assays of SALEiCas9-RIT1 ${ }^{\mathrm{M} 90 \mathrm{I}}+\mathrm{YAP1}^{5 \mathrm{SA}}$ cells transduced with nontargeting control sgRNAs (sgNTC) or RIT1-, AURKA-, IGF1R, or FURIN-targeting sgRNAs ( $n=8$ tumors per sgRNA). Cells were pre-treated with doxcycyline in vitro for 5 days and re-induced at day 26 (arrow) in vivo with doxycycline-containing chow. ${ }^{\star} p<0.05$ by unpaired two-tailed t-test. Source data are provided as a Source Data file.

remains to be fully elucidated, these data suggest that RIT1 ${ }^{\mathrm{M} 90 \mathrm{I}}$ on its own does not trigger YAP1 target transcription but may instead enhance activation of YAP1 targets in settings of YAP1 activation, such as tumors with Hippo pathway loss.

\section{Discussion}

Activation of the RTK-RAS signaling pathway is a near-universal hallmark of lung adenocarcinoma ${ }^{1}$, and drug-targetable mutations identified within the pathway have been harnessed to develop genotype-guided therapies. While targeted therapies sometimes result in exceptional tumor responses and have improved lung cancer patient survival, selection for tumor cell adaptation occurs rapidly, leading to acquired resistance and necessitating treatment with second- and third-line targeted agents until eventual treatment failure ${ }^{82}$. Highlighting the key requirement for activation of RTK-RAS signaling, acquired resistance usually occurs via second-site mutations in the original oncogene or bypass activation of alternative signaling molecules in the network. Although each mutated oncogene in this network shares the ability to activate this pro-tumorigenic cellular signaling pathway, the overlapping but distinct signaling mechanisms of each protein create unique cellular vulnerabilities.

The role of RIT1 activation is a less well-understood part of the RTK-RAS signaling pathway in lung cancer. In this work, we used genetic dependency mapping to uncover the shared and distinct functional requirements of oncogenic variants of EGFR, KRAS, and RIT1. An advantage of our experimental model system was the specific reliance of the cells on the activity of the expressed oncogene in erlotinib, a phenotype firmly linked to each introduced mutation. By intersecting the common dependencies across isogenic cell lines, we were able to exclude pan-essential genes and genes that generally alter sensitivity/resistance to EGFR inhibition, an approach similar to the Daisy Model of gene essentiality ${ }^{83}$. This strategy allowed us to overcome the challenging lack of patient-derived experimental model systems in which to study mutant RIT1 function. A disadvantage of the experimental system, however, was its reliance on treatment with erlotinib, which would not typically be used for the treatment of RIT1-mutant lung cancers. It was therefore critical to validate the dependencies in other cell models in the absence of erlotinib. To this end, we were fortunate to identify a model of RIT1-mutant tumorigenesis through our identification of RIT1/YAP1 synergy in the transformation of human lung epithelial cells (Fig. 7g-l).

RIT1's involvement in Noonan syndrome and lung adenocarcinoma has confirmed its role in the RAS signaling pathway, although the specific mechanism of its function in this pathway has remained elusive. Although RIT1 is reported to physically interact with C-Raf ${ }^{15}$, its ability to activate ERK appears limited compared to RAS and is cell-type dependent (Fig. 1a, d and ref. ${ }^{15}$.). In contrast, oncogenic RAS has been studied for decades and is thought to act downstream of the SHP2/PTPN11 phosphatase and SOS1 guanine nucleotide exchange factor to activate effectors including RAF, PI3K, and RalGDS ${ }^{84}$. In agreement with this model, our data show that RAS-mutant cells are insensitive to knockout of SOS1 and PTPN11 (Fig. 4a). However, other studies suggest that inhibition of PTPN11 can suppress RAS-driven tumorigenesis ${ }^{42,85}$, so further research is required to understand the cell types and contexts where PTPN11 inhibition may be effective. Surprisingly, we find that RIT1 ${ }^{\mathrm{M} 90 \mathrm{I}}$ differs from KRASG12V in its dependency on these factors as well as on the SHOC2 scaffolding protein involved in RAF activation (Fig. 4a). RIT1-mutant cells depend on PTPN11, SOS1, and SHOC2, while showing no requirement for KRAS itself. Likewise, in KRASmutant cells, RIT1 is not required, suggesting KRAS and RIT1 play distinct roles in the regulation of this pathway. An additional difference between RIT1- and KRAS-mutant cells is the continued reliance of RIT1-mutant cells on RTK complex proteins such as GRB2 and IGF1R. Combined with the requirement for PTPN11 and SOS1, we speculate that RIT1 ${ }^{\mathrm{M} 90 \mathrm{I}}$ may be involved in the activation of RTKs themselves, or drive feedback signaling to RTKs. Further studies are needed to clearly define the structure of RIT1's requirement for these proteins.

Beyond these classic RTK-RAS signaling components, we uncovered a surprising vulnerability of RIT1-mutant cells to perturbation of mitotic regulators, particularly components of the spindle assembly checkpoint. We found that RIT1-mutant cells showed heightened sensitivity to loss of mitotic regulators such as AURKA, USP9X, MAD2L1BP, and PLK1, whether by genetic inactivation or small-molecule inhibition, despite no differences in cell proliferation or mitotic index. We showed that RIT1 ${ }^{\mathrm{M} 90 \mathrm{I}}$ weakens the spindle assembly checkpoint, leaving the cells vulnerable to Aurora kinase inhibitors. While our manuscript was in review, a preprint was posted showing the discovery of RIT1 as a MAD2-binding protein that inhibits the mitotic checkpoint complex to accelerate mitotic timing 86 . Using a similar mitotic timing assay to that presented here, they showed that oncogenic RIT1 ${ }^{\text {M90I }}$ accelerates mitosis in U2-OS and HeLa cells. One difference was that the assay was performed in nocodazole; otherwise, the result is nearly identical to the data we show in HeLa cells, suggesting that regulation of mitotic timing and induction of mitotic errors are a general function of oncogenic RIT1. The study, now published ${ }^{87}$, also showed that wild-type RIT1 participates in the spindle assembly checkpoint and that knockout of endogenous RIT1 extends mitotic timing in multiple cell types. Therefore, RIT1 normally participates in the spindle assembly checkpoint and pathogenic levels of RIT1 ${ }^{\mathrm{M} 90 \mathrm{I}}$ alter this normal regulation. The results of our study further imply that this mitotic phenotype confers a targetable vulnerability to RIT1-mutant cells, particularly 
a
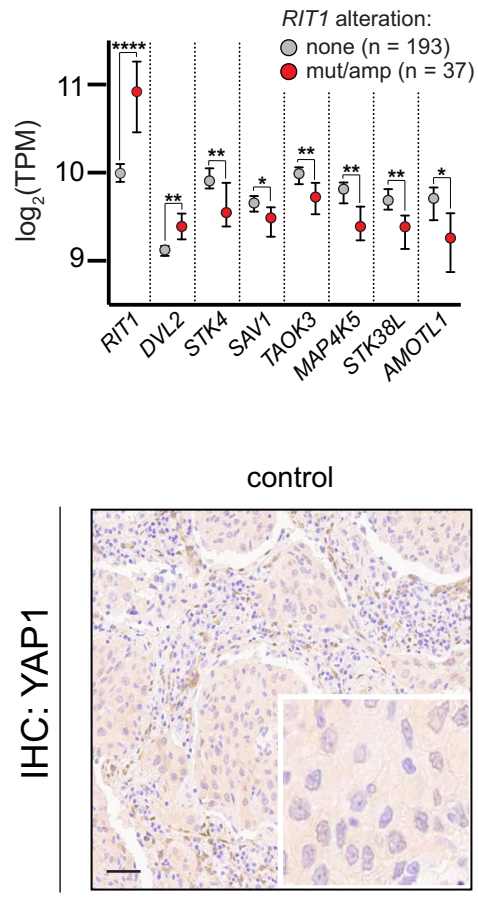

b

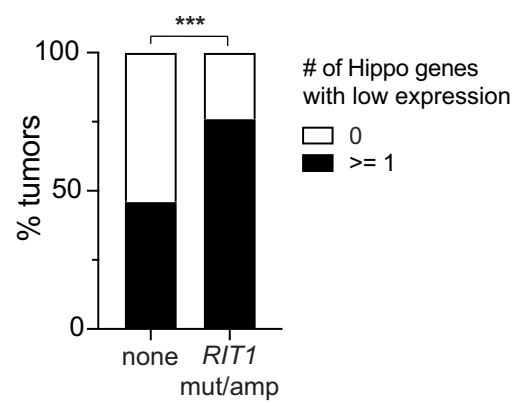

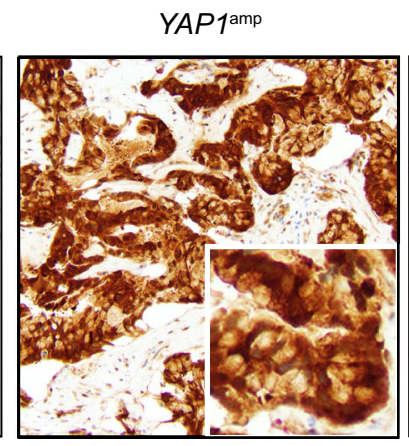

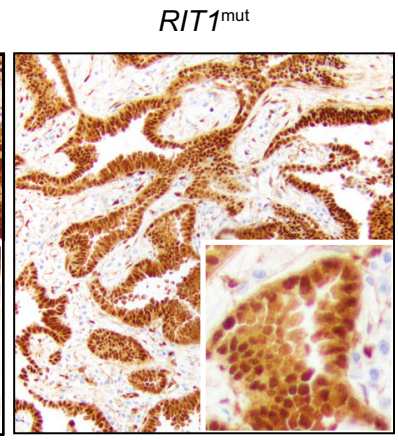

d
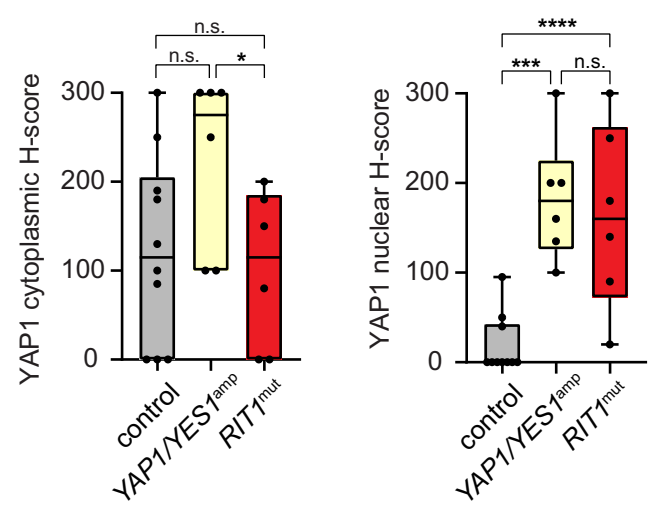

Fig. 8 Hippo pathway loss and YAP1 nuclear overexpression in RIT1-mutant human lung tumors. a RNA-sequencing data of human lung adenocarcinomas from TCGA. Data shown are the median $\pm 95 \%$ confidence interval of $\log _{2}$-transformed transcripts per million (TPM) in RIT1-altered (amplified or mutated, $n=37)$ tumors compared to RIT1 non-altered tumors $(n=193)$. ${ }^{\star} p<0.05,{ }^{\star \star} p<0.01,{ }^{\star \star \star \star} p<0.0001$ by unpaired two-tailed t-test (RIT1: ${ }^{\star \star \star \star} p=1.84 \mathrm{e}-11, \mathrm{DVL2}:{ }^{\star \star} p=0.0025$, STK4: ${ }^{\star \star} p=0.0011$, SAV1: ${ }^{\star} p=0.0278$, TAOK3: ${ }^{\star \star} p=0.0052$, MAP4K5: ${ }^{\star \star} p=0.0023$, STK38L:

${ }^{\star \star} p=0.0023$, AMOTL1: $\left.{ }^{*} p=0.0384\right)$. b Proportion of control or RIT1 amplified or mutated (mut/amp) tumors with low expression of any one Hippo pathway gene; see Methods. ${ }^{\star \star \star} p=0.0006$ by one-tailed Fisher's exact test. c Representative low magnification images and inset zoom of YAP1 IHC in human lung tumor samples, control, YAP1-amplified, and RIT1-mutant, scale bar $=100 \mu \mathrm{m} . n=6-10$ tumors per condition were analyzed $\mathbf{d}$ H-score quantification of control, YAP1/YES1-amplified, and RIT1-mutant human lung tumor samples (Supplementary Data 8 ). $\mathrm{H}$-Score $=\%$ of positive cells multiplied by intensity score of 0-3; see Methods. $n=6-10$ tumors per condition were analyzed. Box plots show the median (center line), first and third quartiles (box edges), and the min and max range (whiskers). n.s., $p>0.05,{ }^{\star} p=0.046,{ }^{\star \star \star} p=0.0009,{ }^{\star \star \star \star} p<0.0001$ by unpaired two-tailed t-test. Source data are provided as a Source Data file.

in the context of Aurora kinase inhibition. Continued studies should investigate additional cell line and cancer models to understand the basis and prevalence of $\mathrm{RIT}^{\mathrm{M} 90 \mathrm{I}}$-driven alisertib sensitivity. Future genotype-directed clinical trials could determine if patients with RIT1-mutant tumors would uniquely benefit from treatment with Aurora kinase inhibitors or other modulators of mitosis. Interestingly, Aurora A activation has been found to drive resistance to the third-generation EGFR inhibitor osimertinib ${ }^{88}$. It is possible that RIT1 ${ }^{\mathrm{M} 90 \mathrm{I}}$ is harnessing this same mechanism to drive erlotinib resistance and cellular transformation.
One of the strongest genetic interactions identified was the pronounced synergy between loss of Hippo pathway genes and RIT1 mutation. The ability of activated YAP1 or NF2 loss to cooperatively transform human lung epithelial cells with RIT1 ${ }^{\text {M90I }}$ (Fig. 7g, h) suggests that this synergy occurs not only in the context of drug resistance, but more generally in oncogenesis. Our analysis of human tumor data showed that over $75 \%$ of RIT1-altered lung adenocarcinomas harbor co-occurring loss of expression of at least one Hippo pathway gene, and we verified high rates of YAP1 nuclear staining in an independent RIT1- 
mutant tumor cohort. While the precise mechanism underlying this synergy remains to be determined, multiple lines of evidence suggest that RIT1 ${ }^{\mathrm{M} 90 \mathrm{I}}$ and YAP1 may be acting independently to cooperatively transform cells. First, mutant RIT1 did not directly stimulate a YAP1 transcriptional signature in RNA-seq of RIT1 $^{\text {M90I }}$-expressing cells (Supplementary Fig. 6d). Second, we observed strong positive selection for loss of upstream Hippo pathway genes; if RIT1 was already promoting Hippo inactivation or YAP1 activation, this cooperation would not be expected. We observed this cooperative effect across multiple different models and phenotypes: loss of NF2 synergized with RIT1 ${ }^{\mathrm{M} 90 \mathrm{I}}$ in the PC9 erlotinib resistance assay and promoted tumor formation of human SALE cells in vivo. The latter phenotype was also conferred by YAP1 ${ }^{5 \mathrm{SA}}$, suggesting that the effect of Hippo suppression is to promote nuclear localization of YAP1. The finding of nuclear YAP1 staining in human RIT1-mutant tumors suggests that RIT1 variants may be most oncogenic in the context of YAP1 activation, and therefore targeting YAP/TEAD activity should be explored as a strategy to suppress RIT1-mutant lung cancer. Future efforts to dissect the mechanism of RIT1/YAP synergy and determine the sensitivity of RIT1-mutant cells to YAP inhibitors should provide further insight into the cooperation between these two oncogenes.

More broadly, our work demonstrates the utility of genomewide CRISPR screens in isogenic cell lines to identify oncogene dependencies and discover therapeutic targets. Isogenic cell systems are particularly valuable to identify critical dependencies of oncogenes that are mutated in $<5 \%$ of cases and consequently not well-represented in CRISPR screening databases such as DepMap ${ }^{22,89}$. The use of deeply characterized phenotypes that are closely linked to and predictive of oncogene function is essential. These directed approaches will complement the analysis of larger cell line panels towards the goal of building genome-scale dependency maps for all human oncogenes.

\section{Methods}

Cell Lines. PC9 cells were a gift from Dr. Matthew Meyerson (Broad Institute) and NCI-H2110 cells were obtained from ATCC (CRL-5924). PC9 and NCI-H2110 were cultured in RPMI-1640 (Gibco) supplemented with 10\% Fetal Bovine Serum (FBS). NIH3T3 cells were obtained from ATCC (CRL-1658), and HeLa-H2B cells were a gift from Dr. Daphne Avgousti (Fred Hutchinson Cancer Research Center) and were originally acquired from Millipore (SCC117). HEK293T cells were obtained from ATCC (CRL-3216). HEK293T, NIH3T3, and HeLa-H2B cells were cultured in Dulbecco's Modified Eagle's Medium (DMEM, Genesee Scientific) supplemented with $10 \%$ FBS (Sigma). AALE and SALE human lung epithelial cells are immortalized with hTERT and the early region of SV40 and were a gift of Dr. William Hahn (Dana-Farber Cancer Institute) and were cultured in small airway epithelial growth media (SAGM) with SAGM supplements and growth factors (PromoCell or Lonza). All cells were maintained at $37^{\circ} \mathrm{C}$ in $5 \% \mathrm{CO} 2$ and confirmed mycoplasma-free.

Lentivirus production. Lentivirus was produced using standard triple transfection protocols: HEK293T cells were co-transfected with lenti-vector (pLKO/PLX303/ PLX317/PXPR003), pCMV-VSV-G (Addgene no. 8454) and psPAX2 (Addgene no. 12260) in TransIT-LTI (Mirus Bio) and OptiMEM (Thermo Fisher Scientific). $18 \mathrm{~h}$ post-transfection, media was changed to high-serum DMEM (30\% FBS). Lentivirus was harvested $48 \mathrm{~h}$ post-transfection.

Vector construction and cell line generation. For NIH3T3 signaling analysis and soft agar assays, the following plasmids were obtained from Addgene; pDONR223HRASG12V (Addgene no. 82090), pDONR223-EGFR ${ }^{L 858 R}$ (Addgene no. 82906), pDONR223-KRASG12V (Addgene no. 81665), and pBabe-puro-Myr-FLAG-AKT1 (Addgene no. 15294). pBabe-puro RIT1 plasmids were generated in a former study ${ }^{7}$. Isogenic NIH3T3 were generated by retroviral transduction and selection with $2 \mu \mathrm{g} / \mathrm{ml}$ puromycin. Protein expression was confirmed by Western blotting. For cell signaling analysis in SALE and AALE cells, pDONR223-KRASWT (Addgene no. 81751), pDONR223-KRAS G12V, and pDONR223-RIT1 ${ }^{\text {M90I }}{ }^{\text {cDNAs }}$ were recombined into pLX317 lentiviral expression vector and lentivirus was generated as above. SALE and AALE cells were transduced with lentivirus followed by selection with $1.5 \mu \mathrm{g} / \mathrm{ml}$ puromycin. Protein expression was confirmed by Western blotting.
For the whole-genome CRISPR knockout screen, PC9 cells stably expressing Cas9 were generated by transducing cells with Cas9 pXPR_111 lentivirus (Genetic Perturbation Platform, Broad Institute) and selecting with blasticidin for 5-7 d. Cas 9 protein expression was determined by Western blot. To determine Cas 9 activity, PC9-Cas9 cells were transduced with a pXPR_011-sgEGFP (Addgene no. 59702), a vector encoding both GFP and a sgRNA targeting GFP. Following selection with puromycin cells were expanded for 3 days. In parallel, untransfected parental PC9 cells and parental PC9 cells transfected with only pXPR_011-sgEGFP were maintained and used as controls for non-GFP expressing and GFP expressing cells, respectively. GFP expression was analyzed in all three cell lines by flow cytometry and data was analyzed using FlowJo software v10.6.2 (Tree Star Inc, Stanford).

To generate isogenic PC9-Cas9 cell lines, pDONR233 vectors encoding EGFRT790M/L858R (Addgene no. 82914), KRASG12V (Addgene no. 81665), RIT1 ${ }^{\text {M90I }}$ (described above), and Firefly luciferase (Addgene no. 25894) were subcloned into the pLX317 lentiviral expression vector. Following verification by Sanger sequencing and restriction digest, stable PC9-Cas9 isogenic cell lines were generated by lentiviral transduction in the presence of $2 \mu \mathrm{g} / \mathrm{ml}$ polybrene followed by selection with $250 \mu \mathrm{g} / \mathrm{mL}$ hygromycin for $48-72 \mathrm{~h}$. Stable lines were expanded, and protein expression was confirmed by Western blotting.

For the small-molecule screen, PC9-RIT1 ${ }^{\mathrm{M} 90 \mathrm{I}}$ and PC9-KRAS ${ }^{\mathrm{G} 12 \mathrm{~V}}$ were generated by transducing parental PC9 cells with lentivirus generated from pLX317-RIT1 ${ }^{\text {M90I }}$ and pLX317-KRAS ${ }^{\text {G12V }}$ generated as described above.

To generate $\mathrm{H} 2110 \mathrm{iCas} 9$ cells, NCI-H2110 cells were transduced with lentivirus generated from Lenti-iCas9-neo (Addgene no. 85400) in the presence of $2 \mu \mathrm{g} / \mathrm{ml}$ polybrene followed by selection with $400 \mu \mathrm{g} / \mathrm{mL}$ G418/Geneticin ${ }^{\mathrm{TM}}$ (ThermoFisher Scientific, 10131035)

To generate isogenic SALEiCas9 cells, parental SALE and SALE-RIT1 ${ }^{\text {M90I }}$ cells were transduced with Lenti-iCas9-neo (Addgene no. 85400) and selected with 400 $\mu \mathrm{g} / \mathrm{mL}$ G418/Geneticin ${ }^{\mathrm{TM}}$ (ThermoFisher Scientific, 10131035). YAP1 ${ }^{5 S A}$ cDNA was PCR amplified from pQCXIH-Myc-YAP1-5SA (Addgene no. 33093) and cloned via Gibson Assembly ${ }^{90}$ into the lentiviral FU-CGW vector ${ }^{91}$ which expresses GFP. SALEiCas9 and SALEiCas9 RIT1 ${ }^{\text {M90I }}$ cells were transduced with YAP $1^{5 S A}$ lentivirus and $48 \mathrm{~h}$ post-transduction GFP expression was confirmed by direct fluorescent expression using the EVOS FL digital inverted microscope Cell Imaging System v1.4 (Thermo Fisher Scientific).

Genome-wide CRISPR gene knockout screen. The human CRISPR Brunello lentiviral pool was obtained from the Broad Institute Genetic Perturbation Platform and is also available from Addgene (73179-LV). The library contains 76,441 sgRNAs targeting 19,114 protein-coding genes and 1,000 non-targeting control sgRNAs. For genome-wide CRISPR screening, 320 million PC9-Cas9-

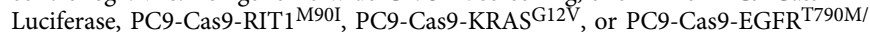
L858R cells were infected with the Brunello Library lentivirus ${ }^{24}$ at a low MOI $(<0.3)$. At $24 \mathrm{~h}$ after infection, the medium was replaced with fresh media containing $1 \mu \mathrm{g} /$ $\mathrm{mL}$ puromycin (Sigma). After selection on day 7, cells were split into 2 or 3 replicates containing 40 million cells each and treated with either DMSO (SigmaAldrich) or $40 \mathrm{nM}$ erlotinib (Selleckchem). Cells were then passaged every 3 days and maintained at 500 -fold coverage. For early time point analysis (day 7) an initial pool of 60 million cells was harvested for genomic DNA extraction from each of the cell lines. After $\sim 12$ doublings, a final pool of 60 million cells was harvested in ice-cold PBS and stored at $-80^{\circ}$.

Genomic DNA was extracted using the QIAamp DNA Blood Maxi Kit (QIAGEN) and the sgRNAs from each sample were PCR amplified by dividing gDNA into multiple $100 \mu \mathrm{l}$ reactions containing a maximum of $10 \mu \mathrm{g}$ gDNA (as recommended by Broad Institute standard protocols). Per 96-well plate, a master mix consisted of $150 \mu \mathrm{l}$ ExTaq polymerase (Takara Bio), $1000 \mu \mathrm{l}$ of 10x ExTaq buffer (Takara Bio), $800 \mu \mathrm{l}$ of dNTP (Takara Bio), $50 \mu \mathrm{l}$ of P5 primer (stock at 100 $\mu \mathrm{M}$ concentration), and $2,075 \mu \mathrm{l}$ water. Each well consisted of $50 \mu \mathrm{l}$ gDNA plus water, $40 \mu \mathrm{l}$ PCR master mix, and $10 \mu \mathrm{l}$ of P7 primer (stock at $5 \mu \mathrm{M}$ concentration). Primer sequences are listed in Supplementary Table 3. PCR cycling conditions: an initial $5 \mathrm{~min}$ at $95^{\circ} \mathrm{C}$; followed by $30 \mathrm{~s}$ at $95^{\circ} \mathrm{C}, 30 \mathrm{~s}$ at $53^{\circ} \mathrm{C}, 20 \mathrm{~s}$ at $72{ }^{\circ} \mathrm{C}$, for 28 cycles; and a final $10 \mathrm{~min}$ extension at $72^{\circ} \mathrm{C}$. PCR samples were purified with Agencourt AMPure XP SPRI beads (Beckman Coulter). Samples were sequenced on a HiSeq 2500 (Illumina). Raw FASTQ files were demultiplexed and sgRNA counts were calculated using PoolQ v2.2.0.

CRISPR validation library and screening. For secondary screening, we generated a custom library containing 1000 non-targeting control sgRNAs and

11,333 sgRNAs targeting 1288 protein-coding genes (Supplementary Data 5). For validation screening, 180 million PC9-Cas9-RIT1 ${ }^{\text {M90I }}$ were infected with the validation library lentivirus at a low MOI $(<0.3)$. At $24 \mathrm{~h}$ after infection, the media was replaced with fresh media containing $1 \mu \mathrm{g} / \mathrm{mL}$ puromycin (Sigma). After selection on day 7 , cells were split into 3 replicates containing 6.6 million cells each and treated with either DMSO (Sigma-Aldrich) or $40 \mathrm{nM}$ erlotinib (Selleckchem). Cells were then passaged every 3 days and maintained at 500-fold coverage. For early time point analysis (day 7) an initial pool of 20 million cells were harvested for genomic DNA extraction from each of the cell lines. After $\sim 12$ doublings, a final pool of 20 million cells were harvested for genomic DNA extraction using the 
QIAamp DNA Blood Maxi Kit (QIAGEN). PCR and sequencing were carried out as described above.

Single and pooled gene knockout generation and genotyping. To induce RIT1, NF2, USP9X, AURKA, or SHOC2 gene knockout in PC9-Cas9-RIT1 ${ }^{\text {M90I }}$ cells, a vector-free CRISPR-mediated editing approach was used: cells were co-transfected using lipofectamine CRISPR max (Life technologies) with three gene-specific synthetic guide RNAs (Synthego, Supplementary Table 1).

To generate RIT1, AURKA, FURIN, and IGF1R, gene knockout in H2110iCas9 and SALEiCas9 cells, sgRNAs (Supplementary Table 2) were cloned into pLentiGuide-Puro (Addgene no. 52963). Lentivirus for each sgRNA was generated as described above. H2110iCas9 were transduced with sgRNA lentivirus in the presence of $2 \mu \mathrm{g} / \mathrm{ml}$ polybrene followed by selection with puromycin $(2 \mu \mathrm{g} / \mathrm{mL})$. SALEiCas9 cells were transduced with sgRNA lentivirus followed by selection with $1.5 \mu \mathrm{g} / \mathrm{ml}$ puromycin.

For each single-gene knockout cell line, gene editing was confirmed by Sanger sequencing. Custom oligos flanking the targeted sites were used to amplify genomic DNA from pooled edited cells (Supplementary Tables 4,5) using High-Fidelity $2 \times$ Master Mix (New England Biolabs). Indel frequencies were quantified by comparing unedited control and knockout cell lines using Inference of CRISPR Edits (ICE) ${ }^{56}$

Proliferation assay. $\mathrm{H} 2110 \mathrm{iCas} 9$ cells expressing either RIT1, AURKA, FURIN, or $I G F 1 R$ sgRNA or non-targeting control sgNTC were seeded in triplicate in 6-well dishes at a density of $0.25 \times 10^{6}$ cells per well. After $24 \mathrm{~h}$ the media was replaced and supplemented with $1 \mu \mathrm{g} / \mathrm{ml}$ of doxycycline to induce Cas 9 expression. At this point, cells were counted and passaged every $3 \mathrm{~d}$ and replated at a density of $.25 \times$ $10^{6}$ cells per well. For Indel analysis and protein expression cell pellets were collected on day 2 and day 5 for gDNA and protein extraction.

Cell lysis and immunoblotting. Whole-cell extracts for immunoblotting were prepared by incubating cells on ice in RTK lysis buffer [ $20 \mathrm{mM}$ Tris ( $\mathrm{pH} 8.0$ ), 2 mM EDTA (pH 8), $137 \mathrm{mM} \mathrm{NaCl}, 1 \%$ IGEPAL CA-630, 10\% Glycerol] plus phosphatase inhibitors (Roche) and protease inhibitors (cOmplete, Mini, EDTA free, Roche) for $20 \mathrm{~min}$. Following centrifugation $(>16,000 \mathrm{~g}$ for $15 \mathrm{~min}$ ), protein lysates were quantified using the Pierce BCA Protein Assay Kit (Thermo Fisher Scientific). Lysates were separated by SDS-PAGE and transferred to nitrocellulose or PVDF membranes using the Trans-blot Turbo Transfer System (BioRad) or iBlot (Invitrogen). Membranes were blocked in 1x Tris Buffered Saline (TBS) with $1 \%$ Casein (BioRad) for $1 \mathrm{~h}$ at room temperature followed by overnight incubation at $4{ }^{\circ} \mathrm{C}$ with primary antibodies diluted in blocking buffer. StarBright (BioRad) or IRDye (LiCOR) secondary antibodies were used for detection and were imaged on ChemiDoc MP Imaging System (BioRad). Loading control and experimental protein(s) were probed on the same membrane unless indicated otherwise. For clarity, loading control is cropped and shown below experimental condition in all panels regardless of the relative molecular weights of the two proteins.

Primary antibodies used for immunoblotting: Phospho-p44/42 MAPK 1:5001:1000 (Erk1/2) (Thr202/Tyr204) (Cell Signaling Technology, 4370), p44/42 MAPK 1:500-1:1000 (Erk1/2) (Cell Signaling Technology, 9107), Phospho-MEK1/2 1:500-1:1000 (Ser217/221) (Cell Signaling Technology, 9154), MEK1 1:500-1:1000 (Cell Signaling Technology, 2352), Phospho-AKT 1:500-1:1000 (Cell Signaling Technology, 4060), AKT 1:500 (Cell Signaling Technology, 2920), EGFR 1:500 (Cell Signaling Technology, 2239), $\beta$-Actin 1:1000 (Cell Signaling Technology, 4970), Vinculin 1:1500 (Sigma, V9264); Firefly Luciferase (Abcam, Ab16466), RIT1 1:1500 (Abcam, Ab53720), KRAS 1:500 (Sigma, WH0003845M1), NF2 1:1000 (Abcam, Ab109244), USP9X 1:1000 (Proteintech, 55054-1-AP), SHOC2/Sur-8 1:100 (Santa Cruz Biotechnology, sc-514779) IGF1R 1:1000 (Cell Signaling Technology, 9750), FURIN 1:500 (Thermo Scientific, PA1-062) and AURKA 1:500 (Cell Signaling Technology, 4718).

Drug treatment and proliferation analysis. For proliferation assays cells were plated in 384-well plates at a density of 800 cells per well in $40 \mu \mathrm{l}$ total volume. One day later, a serial dilution of each inhibitor was performed using a D300e dispenser (Tecan). $96 \mathrm{~h}$ post-treatment, cell viability was determined using CellTiterGlo reagent (Promega) and luminescence quantified on an Envision MultiLabel Plate Reader (PerkinElmer). To calculate the fraction cell viability drug-treated cells were normalized to average cell viability of DMSO-only treated cells. Curve fitting was performed using GraphPad Prism v9.1.1 four-parameter inhibitor response with variable slope. AUC values were calculated by GraphPad Prism v9.1.1. Inhibitors were obtained from SelleckChem: Erlotinib-OSI-744 (S1023), OsimertinibAZD9291 (S7297), Torin 1 (S2827), Alisertib-MLN8237 (S1133), BarasertibAZD1152 (S1147). DMSO (Sigma-Aldrich).

Small-molecule drug screen. PC9-RIT1 ${ }^{\mathrm{M} 90 \mathrm{I}}$ and PC9-KRAS ${ }^{\mathrm{G} 12 \mathrm{~V}}$ were plated in 384 -well plates at a density of 800 cells per well in $40 \mu$ total volume. One day after seeding, cells were treated with $500 \mathrm{nM}$ erlotinib in combination with the smallmolecule library (a kind gift of Dr. Stuart Schreiber, Broad Institute). Each small molecule was tested across an 8-point dilution series (Supplementary Data 6). 96hours post-treatment cell viability was determined using CellTiterGlo reagent
(Promega) and luminescence quantified on an Envision MultiLabel Plate Reader (PerkinElmer). To calculate the fraction of cell viability, drug-treated cells were normalized to $500 \mathrm{nM}$ erlotinib only treated cells. Dose-response curves were plotted using GraphPad Prism v9.1.1; AUC values were generated using GraphPad Prism v9.1.1 and three-parameter inhibitor response setting. Delta-AUC was calculated by subtracting the AUC for each compound in RIT1 ${ }^{\text {M90I }}$ from the AUC of the same compound in KRAS ${ }^{\mathrm{G} 12 \mathrm{~V}}$ cells. Each experiment was carried out in two technical replicates.

Soft agar assays. For soft agar assay, $5 \times 10^{3} \mathrm{NIH} 3 \mathrm{~T} 3$ cells expressing RIT1 ${ }^{\mathrm{M} 90 \mathrm{I}}$ HRAS ${ }^{\mathrm{G} 12 \mathrm{~V}}$, or control (empty vector) were suspended in $1 \mathrm{ml}$ of $0.33 \%$ select agar in DMEM/FBS and plated on a bottom layer of $0.5 \%$ select agar in DMEM/FBS in six-well dishes. Each cell line was analyzed in triplicate. Colonies were photographed after 14-21 days and quantified using CellProfiler v3.092. For soft agar inhibitor experiments, alisertib (MLN8237) or barasertib (AZD1152) was suspended in the top agar solution at a final concentration of $0.01-10 \mu \mathrm{M}$.

In vivo xenograft studies and ethical approval. All animal experiments were carried out with approval by and in accordance with the ethical guidelines of the Fred Hutchinson Cancer Research Center Institutional Animal Care and Use Committee (Protocol \#50967, PI: Berger). Experiments were performed in the Fred Hutch Comparative Medicine facility, which is fully accredited by the Association for Assessment and Accreditation of Laboratory Animal Care (AAALAC) and complies with all United States Department of Agriculture (USDA), Public Health Service (PHS), Washington State and local area animal welfare regulations. Data and methods are reported here in accordance with ARRIVE guidelines ${ }^{93}$. All animals were housed in individually ventilated and HEPA-filtered microisolator cage environments (Allentown Inc. \& Tecniplast) using reusable caging that is autoclaved prior to use and the Steam Plant Facility uses sterile, disposable caging. Additional housing information is as follows: light cycle 12:12 light: dark, temperature $72^{\circ} \pm 3 \mathrm{~F}$, and humidity $30-70 \%$. All animal feed and cage enrichment material is sterilized, and only purified, acidified water is provided. Husbandry is done in height adjustable HEPA-filtered laminar flow cabinets using high-level disinfectants. Athymic nude (nu/nu) mice (4 to 6 weeks old males) for the xenograft study were obtained from Jackson Laboratory, Bar Harbor, ME, USA and allowed to acclimate to the facility for at least 1 week. Due to a supplier shortage, females were not available for use. For SALE doxycycline-inducible Cas9 studies in RIT1 ${ }^{\mathrm{M} 90 \mathrm{I}}+\mathrm{YAP}^{5 \mathrm{SA}}$ cells (Fig. 7i-l), cells were grown in culture and treated with $1 \mu \mathrm{g} / \mathrm{ml}$ doxycycline for five days prior to subcutaneous injection into the flanks of immunocompromised (NU/J) mice. SALE-iCas9 cells expressing sgNF2, RIT1 ${ }^{\text {M90I }}$ + sgNF2, RIT1 ${ }^{\mathrm{M} 90 \mathrm{I}}+$ YAP1 $^{\text {5SA }}+$ sgNTC, sgRIT1, sgAURKA, sgIGF1R, or sgFURIN were harvested by trypsinization, washed in PBS and resuspended at $10^{6}$ cells $/ \mathrm{ml}$ in PBS. Two hundred microliters $\left(2 \times 10^{6}\right.$ cells $)$ were injected into each injection site, $n=2$ injection sites/mouse; 4 mice/condition for 8 experimental replicates per cell line where each experimental unit is a single tumor/injection site. The sample size was chosen to give $98 \%$ power to detect a difference in means of $10 \%$ assuming standard deviation of $5 \%$, or $98 \%$ power to detect a difference in means of $20 \%$ given a standard deviation of $10 \%$. There were no exclusion or inclusion critera; all data points were used for analysis. Animals were not randomized; to avoid error animals within the same cage were injected with the same cell solution and likewise received the same cell solution at each site. Investigators were not blinded to the experimental group information. Cells were allowed to engraft for 1 week, then tumors were measured every 2-3 days using a digital caliper (VWR, Radnor, PA, USA). At day 26, mice were put on doxycycline chow ad libitum $(625 \mathrm{ppm})$ and tumors were measured every 2-3 days until the largest tumor reached $2 \mathrm{~cm}$ in diameter. Tumor volume was calculated with the formula $0.5 \times L \times \mathrm{W}^{2}$ where $L$ is the longest diameter and $W$ is the diameter perpendicular to $L$. Data were analyzed in GraphPad Prism (v9.1.1) and statistical significance was determined by multiple unpaired two-tailed $t$-tests at each time point for each experimental group compared to the sgNTC control with no multiple hypothesis correction and a $\mathrm{p}$ value less than 0.05 considered significant.

\section{Mitotic timing and chromosomal aberration analysis. $\mathrm{RIT1}^{\mathrm{M} 90 \mathrm{I}}$-expressing}

HeLa H2B-GFP cells were generated by transduction with a pLX303-RIT1 ${ }^{\text {M90I }}$ lentivirus and selection with puromycin. Protein expression was confirmed by Western blotting. One day before imaging, cells were seeded at a density of 30,000 cells per well of an 8-well Ibidi glass-bottomed plate. For drug-treated populations, $0.5 \mu \mathrm{M}$ reversine (Selleckchem) or $1 \mu \mathrm{M}$ alisertib was added two hours before imaging. Live-cell imaging was performed using a $\times 20 / 0.70$ Plan Apo Leica objective on an automated Leica DMi8 microscope outfitted with an Andor CSU spinning disk unit equipped with Borealis illumination, an ASI automated stage with Piezo Z-axis top plate, and an Okolab controlled environment chamber (humidified at $37^{\circ} \mathrm{C}$ with $5 \% \mathrm{CO}_{2}$ ). Long-term automated imaging was driven by MetaMorph software (v7.10.0.119). Images were captured with an Andor iXon Ultra 888 EMCCD camera. Images were captured every minute for $18 \mathrm{~h}$. Time in mitosis was measured as time from nuclear envelope breakdown to the onset of anaphase. Imaging experiment was repeated four times with distinct biological replicates and 50 cells were analyzed per cell line per condition. 
Mitotic index was calculated from one time point selected from live-image experiments in HeLa H2B-GFP parental and HeLa H2B-GFP-RIT1 ${ }^{\text {M90I }}$ cells described above. At each time point, three independent, representative images were collected and the mitotic index was calculated based on the number of cells undergoing mitosis (in any phase between prometaphase to telophase) divided by the total number of cells. The same time point was analyzed in two independent experiments for a total of 6 total mitotic index analyses per cell line.

For the mitotic abnormality analysis, HeLa H2B-GFP cells were plated in a 4well Nunc ${ }^{\mathrm{TM}}$ Lab-Tek ${ }^{\mathrm{TM}}$ chamber slide (ThermoFisher Scientific) at a density of 80,000 cells per well. The next day, cells were treated with either DMSO vehicle or $1 \mu \mathrm{M}$ alisertib for $6 \mathrm{~h}$ prior to being washed with 1X PBS and fixed with $4 \%$ paraformaldehyde (from 16\% paraformaldehyde [wt/vol]) in $1 \mathrm{X}$ PBS for $30 \mathrm{~min}$ at RT. Coverslips were mounted with Vectashield antifade mounting medium with 1.5ug/mL DAPI (Vector Laboratories). Slides were analyzed using a $\times 40 / 1.25$ Oil PL Apo Leica objective on a Leica DMi8 outfitted with a TCS SPE scan head with spectral detection. Cells were visualized using the LAS X software platform (v3.5.7.23225). Representative images were captured using a $\times 40 / 1.30$ Plan Apo Leica objective on a Leica DMi8 outfitted with a TCS SPE scan head with spectral detection. Images were acquired using the LAS X software platform (v3.5.5.19976). Images were corrected for brightness and contrast using FIJI (v2.1.0/1.53c). Images are single sections. For each biological replicate, 60 cells were analyzed per cell line. The prevalence of chromosome bridges, lagging and chromosome misalignment, micronuclei, aneuploidy (i.e. notable, unequal separation of chromosomes), polyploidy (i.e. evidence of unsuccessful cytokinesis) and normal separation of chromosomes were recorded.

Gene set enrichment analysis. In Fig. $4 \mathrm{~b}$ and Fig. $7 \mathrm{~d}$, gene set overlap analysis was performed using the MsigDB (v7.4) (4, $^{95}$ and StringDB (v11.0 ${ }^{96}$ databases. For all analyses an FDR cut-off of $<.05$ was used. To account for differences in the number of cooperating factors between RIT1 ${ }^{\mathrm{M} 90 \mathrm{I}}$ and KRAS ${ }^{\mathrm{G} 12 \mathrm{~V}}$, overlap analysis was carried out on the top 152 positively selected genes from each screen. In Supplementary Fig. 6d, GSEA of transcriptome data was completed using preranked gene lists using GSEA 4.1.090 using the oncogenic signature gene sets (c6.all. v7.2.symbols.gmt) containing 189 gene sets. Number of permutations was set to 1000, classic enrichment statistic was used with 15 and 500 set as minimum and maximum, respectively, for set inclusion.

TCGA RNA expression analysis. RIT1 RNA-seq data from TCGA lung adenocarcinomas (230 samples) ${ }^{6}$ was obtained from cBioPortal (v3.6.18) for Cancer Genomics (http://cbioportal.org). The z-score for RIT1 mRNA expression is determined for each sample by comparing RIT1 mRNA expression to the distribution of RIT1 expression in all diploid samples ${ }^{97}$.

For differential gene expression analysis, RIT1-altered samples were defined as tumors with either mutation of RIT1 $(n=5)$ or amplification of RIT1 $(n=32)$. The remaining 193 tumors were considered non-RIT1-altered. Differential expression analysis was performed in cBioPortal, and genes with $q$-value $<=0.25$ were downloaded from cBioPortal (http://cbioportal.org). Pathway enrichment analysis was carried out on the 1015 under-expressed genes identified in RIT1-altered sample using the StringDB ${ }^{96}$ database with an FDR $<.05$.

For analysis of individual Hippo pathway genes in RIT1 altered samples, we included the following genes as Hippo pathway genes: AMOTL1, NF2, STK4, STK38L MAP4K5, TAOK3, SAV1. To determine the portion of RIT1 altered tumors with low expression of any one Hippo pathway gene, low expression was defined as $>1$ standard deviation lower than the mean expression of the 230-sample dataset. Then we determined the proportion of samples with low expression of at least one of the above genes, or with normal expression of all genes.

YAP1 immunohistochemistry in RIT1-mutant human tumors. Epitope retrieval was performed using Leica Bond III ER2 for $30 \mathrm{~min}$ on the stainer, followed by incubation with the YAP1 antibody (Santa Cruz, clone 63.7) for an additional 30 min, and detection using Bond Polymer Refine DAB IHC detection kit. For quantitative assessment of YAP1 labeling in the nuclear and cytoplasmic compartments, a histoscore (H-score) was derived by multiplying percent positivity in tumor cells by the corresponding staining intensity $(1=$ weak, $2=$ moderate, $3=$ strong) within each compartment yielding a range of possible $\mathrm{H}$-scores of $0-300$.

Transcriptome profiling. Three technical replicates per cell line were harvested at $\sim 90 \%$ confluence. Total RNA was extracted from lysed cells using Direct-zol RNA Miniprep plus (Zymo Research). Libraries of $50 \mathrm{bp}$ paired-end reads were constructed using the Illumina TruSeq kit, which utilizes non-strand-specific poly-A selection. Libraries were pooled and sequenced on the Illumina HiSeq 2500 platform (Fred Hutch Genomics Core). Reads were aligned to the human reference genome build hg19/GRCh37 using STAR v2.5.3a $\mathrm{a}^{98}$. Alignments were annotated, reordered, and indexed using Picard Tools v1.114 (http://broadinstitute.github.io/ picard). To ensure sequencing and alignment quality, read statistics for each RNAseq sample were calculated using RseQC v3.0.199, including total library size, number of ribosomal RNA reads, and number of mapped and unmapped read pairs. Transcripts were quantified based on hg19 gene annotations using the featureCounts program included in Subread v1.5.3 ${ }^{100}$. Gene-level CPM and RPKM values were calculated with edgeR v3.30.3 ${ }^{101}$ then converted into transcripts per million values with an in-house script. In total, 12,458 genes were identified with average expression level of at least $0.1 \log _{2} \mathrm{CPM}$ across all samples. Differential expression analyses comparing SALE-RIT1 ${ }^{\mathrm{M} 90 I}$, SALE-YAP1 ${ }^{5 S A}$ or SALERIT1 ${ }^{\mathrm{M} 90 \mathrm{I}}+\mathrm{YAP}^{5 \mathrm{SA}}$ cells against vector control SALE were performed using edgeR v3.30.3 ${ }^{101}$.

RT-qPCR. Total RNA was extracted from three biological replicates of SALE parental, SALE-RIT1 ${ }^{\text {M90I }}$, SALE-YAP1 $1^{5 S A}$ and SALE-RIT1 ${ }^{\text {M90I }}+$ YAP1 $^{\text {5SA }}$ cells as described above. Reverse transcription (RT) was performed with $800 \mathrm{ng}$ RNA and the SuperScript IV First-Strand Synthesis System (Invitrogen). For each quantitative RT-PCR reaction, input cDNA was optimized to the following amounts: 10 $\mathrm{ng}$ for $18 \mathrm{~S}, 40 \mathrm{ng}$ for ITGB2 and COL4A3, and $100 \mathrm{ng}$ for TNNT2 reactions. cDNA was amplified using TaqMan gene expression assays (ThermoFisher Scientific): ITGB2 (assay ID: Hs00164957_m1), TNNT2 (assay ID: Hs00943911_m1), COL4A3 (assay ID: Hs01022502_m1), and 18S (assay ID: Hs99999901_s1). Reactions were run using the BioRad CFX384 Real-Time system. Expression was normalized to $18 \mathrm{~S}$ within each sample in the same experiment, and relative expression was quantified via the $2^{-\Delta \Delta \mathrm{Ct}}$ method. Results are means of three technical replicates. Graphs and statistical analysis were performed in GraphPad Prism v9.1.1.

\section{Statistical analysis}

Genome-wide CRISPR screen. A $\log _{2}$-normalized count matrix of sequencing reads mapped to sgRNAs was used as an input to MAGeCK (v0.5.7) ${ }^{35}$. sgRNAs with $<1$ read-per-million in the sequencing of the Brunello library plasmid were excluded from the dataset. The following sgRNAs were determined to not target the introduced cDNA oncogenes, and so were excluded from the dataset.

KRAS: (AGATATTCACCATTATAGGT) RIT1: (CATGCG GGACCAGTATATGA,GTGATGATCTGGCTTACCAA) EGFR: (TGTCACCACATAATTACCTG)

Reproducibility of the screen was assessed by calculating Pearson correlations of pairwise replicate comparisons for each replicate set $(n=2-3$ replicates per screen arm). Pearson correlations ranged from 0.58 to 0.94 (median $=0.82)$. Scores from early time-point (ETP) samples were highly correlated to plasmid DNA (median $=0.93$; Supplementary Fig. 2e) so comparison to plasmid DNA was used for all subsequent analyses. Guide RNAs were collapsed to gene scores using MAGeCK (v0.5.7), and $\log _{2}$ (fold-change) values (LFC) were computed compared to starting guide RNA abundance in plasmid DNA. To check the performance of each screen, we calculated robust strictly standardized mean difference (SSMD) statistics ${ }^{22}$ for each replicate, comparing the LFC between non-targeting sgRNAs and LFC values of sgRNAs targeting the spliceosomal, ribosomal, and proteasomal gene sets from KEGG ${ }^{102}$. All endpoint replicates passed the quality control threshold of SSMD $<-0.5$, with the median SSMD $=-3.9$ (Supplementary Data 1). Normalization across screens was based on published methods; ${ }^{22}$ first, the LFC data were normalized within each replicate by subtracting the median LFC of the replicate and then dividing by the median average deviation. Next, we scaled each replicate based on the LFC of common essential and nonessential genes (Supplementary Data 10) using previously defined lists by DepMap ${ }^{89}$. The data were scaled such that the median of the nonessential genes in each replicate is 0 and the median of the essential genes is -1 . CRISPR scores were defined as this scaled, normalized LFC data. The final normalized, scaled data are supplied as (Supplementary Data 2). For analysis of expressed vs. non-expressed genes (Supplementary Fig. 2g), we defined expressed genes from RNA-sequencing data of PC9 cells ${ }^{103}$ as genes with $\log _{2}(\mathrm{TPM})>2$ and non-expressed genes as $\log _{2} \mathrm{TPM}<1$.

Statistical tests. Statistical tests are indicated in the figure legends. Results were analyzed for statistical significance with GraphPad 
Prism v9.1.1 or $\mathrm{R}$ (v3.6.3). A $p$-value of $<0.05$ was considered significant $\left({ }^{*} p<0.05,{ }^{* *} p<0.01,{ }^{* * *} p<0.001,{ }^{* * *} p<0.0001\right)$.

Reporting summary. Further information on research design is available in the Nature Research Reporting Summary linked to this article.

\section{Data availability}

The RNA-seq data generated in this study have been deposited in the Gene Expression Omnibus under accession code GSE165631. Analysis of this dataset (Supplementary Fig. 6) is available in Supplementary Data 9. CRISPR screen data (Figs. 2-4, Supplementary Figs. 2-5) generated in this study are provided in Supplementary Data files as follows: sequencing results (Supplementary Data 1), normalized CRISPR scores (Supplementary Data 2), baseline synthetic lethal genes (Supplementary Data 3), oncogene dependencies (Supplementary Data 4), and validation screen analysis (Supplementary Data 5). The small-molecule screen data (Fig. 5) generated in this study are provided in Supplementary Data 6. Pathway analysis of TCGA RNA-seq (Fig. 8a) is provided in Supplementary Data 7. MSK-IMPACT information and H-scores (Fig. 8) is provided in Supplementary Data 8. Lists of common essential and non-essential genes from DepMap.org used for normalization of CRISPR data are provided in

Supplementary Data 10. RNA expression data generated by TCGA were downloaded via cBioPortal for Cancer Genomics from http://www.cbioportal.org/study/summary? id=luad_tcga_pub. Source TCGA data used are available via Open Access at https://gdc. cancer.gov/about-data/publications/pancanatlas. The authors declare that all other data supporting the findings of this study are available within the paper and its Supplementary information files. Source data are provided with this paper, including uncropped gels for all Western blots and underlying data for all figures.

Received: 15 July 2020; Accepted: 12 July 2021;

Published online: 09 August 2021

\section{References}

1. Campbell, J. D. et al. Distinct patterns of somatic genome alterations in lung adenocarcinomas and squamous cell carcinomas. Nat. Genet. 48, 607-616 (2016).

2. Luo, J., Solimini, N. L. \& Elledge, S. J. Principles of cancer therapy: oncogene and non-oncogene addiction. Cell 136, 823-837 (2009).

3. Mok, T. S. et al. Gefitinib or carboplatin-paclitaxel in pulmonary adenocarcinoma. N. Engl. J. Med. 361, 947-957 (2009).

4. Mok, T. S. et al. Osimertinib or platinum-pemetrexed in EGFR T790Mpositive lung cancer. N. Engl. J. Med. 376, 629-640 (2017).

5. Shaw, A. T. et al. Crizotinib versus chemotherapy in advanced ALK-positive lung cancer. N. Engl. J. Med. 368, 2385-2394 (2013).

6. TCGA. Comprehensive molecular profiling of lung adenocarcinoma. Nature 511, 543-550 (2014).

7. Berger, A. H. et al. Oncogenic RIT1 mutations in lung adenocarcinoma. Oncogene 33, 4418-4423 (2014).

8. Berger, A. H. et al. High-throughput phenotyping of lung cancer somatic mutations. Cancer Cell 30, 214-228 (2016).

9. Lee, C. H., Della, N. G., Chew, C. E. \& Zack, D. J. Rin, a neuron-specific and calmodulin-binding small G-protein, and Rit define a novel subfamily of ras proteins. J. Neurosci. 16, 6784-6794 (1996).

10. Shao, H., Kadono-Okuda, K., Finlin, B. S. \& Andres, D. A. Biochemical characterization of the Ras-related GTPases Rit and Rin. Arch. Biochem. Biophys. 371, 207-219 (1999).

11. McCoach, C. E. et al. Resistance mechanisms to targeted therapies in ROS1+ and ALK+ non-small cell lung cancer. Clin. Cancer Res. 24, 3334-3347 (2018).

12. Gómez-Seguí, I. et al. Novel recurrent mutations in the RAS-like GTP-binding gene RIT1 in myeloid malignancies. Leukemia 27, 1943-1946 (2013).

13. Cherniack, A. D. et al. Integrated molecular characterization of uterine carcinosarcoma. Cancer Cell 31, 411-423 (2017).

14. Aoki, Y. et al. Gain-of-function mutations in RIT1 cause Noonan Syndrome, a RAS/MAPK pathway syndrome. Am. J. Hum. Genet. 93, 173-180 (2013).

15. Castel, P. et al. RIT1 oncoproteins escape LZTR1-mediated proteolysis. Science 363, 1226-1230 (2019).

16. Fang, Z. et al. Biochemical classification of disease-associated mutants of RASlike protein expressed in many tissues (RIT1). J. Biol. Chem. 291, 15641-15652 (2016)

17. Lundberg, A. S. et al. Immortalization and transformation of primary human airway epithelial cells by gene transfer. Oncogene 21, 4577-4586 (2002).

18. Barbie, D. A. et al. Systematic RNA interference reveals that oncogenic KRASdriven cancers require TBK1. Nature 462, 108-112 (2009).

19. Luo, J. et al. A genome-wide RNAi screen identifies multiple synthetic lethal interactions with the Ras Oncogene. Cell 137, 835-848 (2009).
20. Wang, T. et al. Gene essentiality profiling reveals gene networks and synthetic lethal interactions with oncogenic Ras. Cell 168, 890-903.e15 (2017).

21. Puyol, M. et al. A synthetic lethal interaction between K-Ras oncogenes and Cdk4 unveils a therapeutic strategy for non-small cell lung carcinoma. Cancer Cell 18, 63-73 (2010).

22. Meyers, R. M. et al. Computational correction of copy number effect improves specificity of CRISPR-Cas9 essentiality screens in cancer cells. Nat. Genet. 49, 1779-1784 (2017)

23. DeWeirdt, P. C. et al. Genetic screens in isogenic mammalian cell lines without single cell cloning. Nat. Commun. 11, 752 (2020).

24. Doench, J. G. et al. Optimized sgRNA design to maximize activity and minimize off-target effects of CRISPR-Cas9. Nat. Biotechnol. 34, 184-191 (2016).

25. Aguirre, A. J. \& Hahn, W. C. Synthetic lethal vulnerabilities in KRAS-mutant cancers. Cold Spring Harb. Perspect. Med. 8, a031518 (2018).

26. Lin, A. \& Sheltzer, J. M. Discovering and validating cancer genetic dependencies: approaches and pitfalls. Nat. Rev. Genet. 21, 671-682 (2020).

27. O’Neil, N. J., Bailey, M. L. \& Hieter, P. Synthetic lethality and cancer. Nat. Rev. Genet. 18, 613-623 (2017).

28. Yarden, Y. \& Shilo, B.-Z. SnapShot: EGFR signaling pathway. Cell 131, 1018. e1-1018.e2 (2007)

29. Engelman, J. A. Targeting PI3K signalling in cancer: opportunities, challenges and limitations. Nat. Rev. Cancer 9, 550-562 (2009).

30. Dede, M., McLaughlin, M., Kim, E. \& Hart, T. Multiplex enCas12a screens detect functional buffering among paralogs otherwise masked in monogenic Cas9 knockout screens. Genome Biol. 21, 262 (2020).

31. Parrish, P. C. R. et al. Discovery of synthetic lethal and tumor suppressive paralog pairs in the human genome. Cold Spring Harbor Laboratory https:// doi.org/10.1101/2020.12.20.423710. (2020)

32. Oxnard, G. R. et al. Assessment of resistance mechanisms and clinical implications in patients with EGFR T790M-positive lung cancer and acquired resistance to osimertinib. JAMA Oncol. 4, 1527-1534 (2018).

33. Imielinski, M. et al. Mapping the hallmarks of lung adenocarcinoma with massively parallel sequencing. Cell 150, 1107-1120 (2012).

34. Unni, A. M., Lockwood, W. W., Zejnullahu, K., Lee-Lin, S.-Q. \& Varmus, H. Evidence that synthetic lethality underlies the mutual exclusivity of oncogenic KRAS and EGFR mutations in lung adenocarcinoma. Elife 4, e06907 (2015).

35. $\mathrm{Li}, \mathrm{W}$. et al. MAGeCK enables robust identification of essential genes from genome-scale CRISPR/Cas9 knockout screens. Genome Biol. 15, 554 (2014).

36. Hart, T. et al. High-resolution CRISPR screens reveal fitness genes and genotype-specific cancer liabilities. Cell 163, 1515-1526 (2015).

37. Cabodi, S., del Pilar Camacho-Leal, M., Di Stefano, P. \& Defilippi, P. Integrin signalling adaptors: not only figurants in the cancer story. Nat. Rev. Cancer 10, 858-870 (2010).

38. Wang-Rodriguez, J., Dreilinger, A. D., Alsharabi, G. M. \& Rearden, A. The signaling adapter protein PINCH is up-regulated in the stroma of common cancers, notably at invasive edges. Cancer 95, 1387-1395 (2002)

39. Takanami, I. Increased expression of integrin-linked kinase is associated with shorter survival in non-small cell lung cancer. BMC Cancer 5, 1 (2005).

40. Karachaliou, N. et al. Integrin-linked kinase (ILK) and src homology 2 domaincontaining phosphatase 2 (SHP2): Novel targets in EGFR-mutation positive nonsmall cell lung cancer (NSCLC). EBioMedicine 39, 207-214 (2019).

41. Fuchs, B. C. et al. Epithelial-to-mesenchymal transition and integrin-linked kinase mediate sensitivity to epidermal growth factor receptor inhibition in human hepatoma cells. Cancer Res. 68, 2391-2399 (2008).

42. Mainardi, S. et al. SHP2 is required for growth of KRAS-mutant non-smallcell lung cancer in vivo. Nat. Med. https://doi.org/10.1038/s41591-018-0023-9 (2018).

43. Chen, Y.-N. P. et al. Allosteric inhibition of SHP2 phosphatase inhibits cancers driven by receptor tyrosine kinases. Nature 535, 148-152 (2016).

44. Cox, A. D., Der, C. J. \& Philips, M. R. Targeting RAS membrane association: back to the future for anti-RAS drug discovery? Clin. Cancer Res. 21, 1819-1827 (2015).

45. Kim, J. et al. XPO1-dependent nuclear export is a druggable vulnerability in KRAS-mutant lung cancer. Nature 538, 114-117 (2016).

46. Denduluri, S. K. et al. Insulin-like growth factor (IGF) signaling in tumorigenesis and the development of cancer drug resistance. Genes Dis. 2, 13 (2015).

47. Massagué, J. \& Czech, M. P. The subunit structures of two distinct receptors for insulin-like growth factors I and II and their relationship to the insulin receptor. J. Biol. Chem. 257, 5038-5045 (1982).

48. Ward, C. W. et al. The three dimensional structure of the type I insulin-like growth factor receptor. Mol. Pathol. 54, 125-132 (2001).

49. Khatib, A.-M. et al. Inhibition of proprotein convertases is associated with loss of growth and tumorigenicity of HT-29 human colon carcinoma cells. J. Biol. Chem. 276, 30686-30693 (2001).

50. Han, K. et al. CRISPR screens in cancer spheroids identify $3 \mathrm{D}$ growth-specific vulnerabilities. Nature 580, 136-141 (2020).

51. Schwickart, M. et al. Deubiquitinase USP9X stabilizes MCL1 and promotes tumour cell survival. Nature 463, 103-107 (2010). 
52. Peddaboina, C. et al. The downregulation of Mcl-1 via USP9X inhibition sensitizes solid tumors to Bcl-xl inhibition. BMC Cancer 12, 541 (2012).

53. Skowyra, A., Allan, L. A., Saurin, A. T. \& Clarke, P. R. USP9X limits mitotic checkpoint complex turnover to strengthen the spindle assembly checkpoint and guard against chromosomal instability. Cell Rep. 23, 852-865 (2018).

54. Vong, Q. P., Cao, K., Li, H. Y., Iglesias, P. A. \& Zheng, Y. Chromosome alignment and segregation regulated by ubiquitination of survivin. Science 310, 1499-1504 (2005).

55. Cao, J. et al. An easy and efficient inducible CRISPR/Cas9 platform with improved specificity for multiple gene targeting. Nucleic Acids Res. 44, e149 (2016).

56. Hsiau, T. et al. Inference of CRISPR edits from Sanger trace data. bioRxiv https://doi.org/10.1101/251082 (2018).

57. Lara-Gonzalez, P., Westhorpe, F. G. \& Taylor, S. S. The spindle assembly checkpoint. Curr. Biol. 22, R966-R980 (2012).

58. Tsherniak, A. et al. Defining a cancer dependency map. Cell 170, 564-576.e16 (2017).

59. Meraldi, P., Draviam, V. M. \& Sorger, P. K. Timing and checkpoints in the regulation of mitotic progression. Dev. Cell 7, 45-60 (2004).

60. Topham, C. H. \& Taylor, S. S. Mitosis and apoptosis: how is the balance set? Curr. Opin. Cell Biol. 25, 780-785 (2013).

61. Sivakumar, S., Daum, J. R. \& Gorbsky, G. J. Live-cell fluorescence imaging for phenotypic analysis of mitosis. Methods Mol. Biol. 1170, 549-562 (2014).

62. Kanda, T., Sullivan, K. F. \& Wahl, G. M. Histone-GFP fusion protein enables sensitive analysis of chromosome dynamics in living mammalian cells. Curr. Biol. 8, 377-385 (1998).

63. Dobles, M., Liberal, V., Scott, M. L., Benezra, R. \& Sorger, P. K. Chromosome missegregation and apoptosis in mice lacking the mitotic checkpoint protein Mad2. Cell 101, 635-645 (2000).

64. Iwanaga, Y. et al. Heterozygous deletion of mitotic arrest-deficient protein 1 (MAD1) increases the incidence of tumors in mice. Cancer Res. 67, 160-166 (2007).

65. Li, M., Fang, X., Wei, Z., York, J. P. \& Zhang, P. Loss of spindle assembly checkpoint-mediated inhibition of $\mathrm{Cdc} 20$ promotes tumorigenesis in mice. $J$. Cell Biol. 185, 983-994 (2009).

66. Zeng, X., Xu, W. K., Lok, T. M., Ma, H. T. \& Poon, R. Y. C. Imbalance of the spindle-assembly checkpoint promotes spindle poison-mediated cytotoxicity with distinct kinetics. Cell Death Dis. 10, 314 (2019).

67. $\mathrm{Yu}, \mathrm{F}$. et al. Aurora-A promotes the establishment of spindle assembly checkpoint by priming the Haspin-Aurora-B feedback loop in late G2 phase. Cell Discov. 3, 16049 (2017).

68. Courtheoux, T. et al. Aurora A kinase activity is required to maintain an active spindle assembly checkpoint during prometaphase. J. Cell Sci. 131, jcs191353 (2018).

69. Sos, M. L. et al. PTEN loss contributes to erlotinib resistance in EGFR-mutant lung cancer by activation of Akt and EGFR. Cancer Res. 69, 3256-3261 (2009).

70. Liao, S. et al. A genetic interaction analysis identifies cancer drivers that modify EGFR dependency. Genes Dev. 31, 184-196 (2017).

71. Okada, M. Regulation of the SRC family kinases by Csk. Int. J. Biol. Sci. 8, 1385-1397 (2012).

72. Ishizawar, R. \& Parsons, S. J. c-Src and cooperating partners in human cancer. Cancer Cell 6, 209-214 (2004).

73. Meng, Z., Moroishi, T. \& Guan, K.-L. Mechanisms of Hippo pathway regulation. Genes Dev. 30, 1-17 (2016).

74. Lee, J. E. et al. Hippo pathway effector YAP inhibition restores the sensitivity of EGFR-TKI in lung adenocarcinoma having primary or acquired EGFR-TKI resistance. Biochem. Biophys. Res. Commun. 474, 154-160 (2016).

75. Zhao, B. et al. Inactivation of YAP oncoprotein by the Hippo pathway is involved in cell contact inhibition and tissue growth control. Genes Dev. 21, 2747-2761 (2007).

76. Rosenbluh, J. et al. $\beta$-Catenin-driven cancers require a YAP1 transcriptional complex for survival and tumorigenesis. Cell 151, 1457-1473 (2012).

77. Park, H. W. et al. Alternative Wnt signaling activates YAP/TAZ. Cell 162, 780-794 (2015).

78. Zehir, A. et al. Mutational landscape of metastatic cancer revealed from prospective clinical sequencing of 10,000 patients. Nat. Med. 23, 703-713 (2017).

79. Fan, P.-D. et al. YES1 amplification is a mechanism of acquired resistance to EGFR inhibitors identified by transposon mutagenesis and clinical genomics. Proc. Natl Acad. Sci. USA 115, E6030-E6038 (2018).

80. Zanconato, F., Cordenonsi, M. \& Piccolo, S. YAP/TAZ at the roots of cancer. Cancer Cell 29, 783-803 (2016).

81. Cordenonsi, M. et al. The Hippo transducer TAZ confers cancer stem cellrelated traits on breast cancer cells. Cell 147, 759-772 (2011).

82. Rotow, J. \& Bivona, T. G. Understanding and targeting resistance mechanisms in NSCLC. Nat. Rev. Cancer 17, 637-658 (2017).

83. Hart, T., Brown, K. R., Sircoulomb, F., Rottapel, R. \& Moffat, J. Measuring error rates in genomic perturbation screens: gold standards for human functional genomics. Mol. Syst. Biol. 10, 733 (2014).

84. Simanshu, D. K., Nissley, D. V. \& McCormick, F. RAS proteins and their regulators in human disease. Cell 170, 17-33 (2017).
85. Ruess, D. A. et al. Mutant KRAS-driven cancers depend on PTPN11/SHP2 phosphatase. Nat. Med. https://doi.org/10.1038/s41591-018-0024-8 (2018).

86. Cuevas-Navarro, A. et al. The RAS GTPase RIT1 compromises mitotic fidelity through spindle assembly checkpoint suppression. Cold Spring Harbor Laboratory https://doi.org/10.1101/2020.11.17.386177 (2020).

87. Cuevas-Navarro, A. et al. The RAS GTPase RIT1 compromises mitotic fidelity through spindle assembly checkpoint suppression. Curr Biol. https://doi.org/ 10.1016/j.cub.2021.06.030 (2021)

88. Shah, K. N. et al. Aurora kinase A drives the evolution of resistance to third-generation EGFR inhibitors in lung cancer. Nat. Med. 25, 111-118 (2019).

89. Dempster, J. M. et al. Extracting biological insights from the project Achilles Genome-Scale CRISPR screens in cancer cell lines. bioRxiv https://doi.org/ 10.1101/720243 (2019).

90. Gibson, D. G. et al. Enzymatic assembly of DNA molecules up to several hundred kilobases. Nat. Methods 6, 343-345 (2009).

91. Xin, L. et al. Progression of prostate cancer by synergy of AKT with genotropic and nongenotropic actions of the androgen receptor. Proc. Natl Acad. Sci. USA 103, 7789-7794 (2006).

92. McQuin, C. et al. CellProfiler 3.0: next-generation image processing for biology. PLoS Biol. 16, e2005970 (2018).

93. Percie du Sert, N. et al. The ARRIVE guidelines 2.0: Updated guidelines for reporting animal research. PLoS Biol. 18, e3000410 (2020).

94. Subramanian, A. et al. Gene set enrichment analysis: a knowledge-based approach for interpreting genome-wide expression profiles. Proc. Natl Acad. Sci. USA 102, 15545-15550 (2005).

95. Liberzon, A. et al. Molecular signatures database (MSigDB) 3.0. Bioinformatics 27, 1739-1740 (2011).

96. Szklarczyk, D. et al. STRING v11: protein-protein association networks with increased coverage, supporting functional discovery in genome-wide experimental datasets. Nucleic Acids Res. 47, D607-D613 (2019).

97. Gao, J. et al. Integrative analysis of complex cancer genomics and clinical profiles using the cBioPortal. Sci. Signal. 6, 11 (2013).

98. Dobin, A. et al. STAR: ultrafast universal RNA-seq aligner. Bioinformatics 29 15-21 (2013).

99. Wang, L., Wang, S. \& Li, W. RSeQC: quality control of RNA-seq experiments. Bioinformatics 28, 2184-2185 (2012).

100. Liao, Y., Smyth, G. K. \& Shi, W. featureCounts: an efficient general purpose program for assigning sequence reads to genomic features. Bioinformatics $\mathbf{3 0}$, 923-930 (2014).

101. Robinson, M. D., McCarthy, D. J. \& Smyth, G. K. edgeR: a bioconductor package for differential expression analysis of digital gene expression data. Bioinformatics 26, 139-140 (2010).

102. Kanehisa, M. \& Goto, S. KEGG: kyoto encyclopedia of genes and genomes. Nucleic Acids Res. 28, 27-30 (2000).

103. Thomas, J. D. et al. RNA isoform screens uncover the essentiality and tumorsuppressor activity of ultraconserved poison exons. Nat. Genet. 52, 84-94 (2020).

\section{Acknowledgements}

The authors thank Amy Goodale (Broad Institute) for technical assistance in the design and synthesis of the targeted validation screening library and Dr. Daphne Avgousti (Fred Hutchinson Cancer Research Center) for providing HeLa H2B-GFP cells. We thank the Broad Institute Genetic Perturbation Platform for technical assistance and advice and the Fred Hutch Genomics Shared Resource and Comparative Medicine Shared Resource (supported by NIH/NCI Cancer Center Support Grant P30 CA015704) and the Northwest Genomics Center. We thank the Fred Hutch Cellular Imaging Shared Resource (supported by the Fred Hutch/University of Washington Cancer Consortium P30 CA015704) for assistance with microscopy and image analysis. This research was funded in part through NCI R00CA197762 and R37CA252050 to A.H.B, donations from the Smith family to A.H.B., NIH/NCI Cancer Center Support Grant P30 CA015704 New Investigator support to A.H.B., a Lung Cancer Research Foundation Research Grant to A. V., the Hutch United postdoctoral fellowship to A.V. A.R. was supported in part by PHS NRSA T32GM007270 from NIGMS. P.C.R.P. was supported in part by NSF DGE1762114 and E.M.H was supported by NIGMS grant R35GM124766.

\section{Author contributions}

A.H.B. conceived of the project. A.H.B., R.K.B., E.M.H., and M.L. supervised the project. A.V., F.P., E.M.H., M.L., and A.H.B. designed experiments. A.V., N.T.N., A.K.R., P.C.R.P., A.L., M.K.B., A.G., and A.H.B. analyzed the data. A.V., N.N., A.K.R., P.C.R.P., S.K., I.F., J. D.T., J.K.L., F.D., J.C., J.W., M.R., N.R., M.K.B., A.G., J.D.T., and A.H.B. conducted experiments. A.V. and A.H.B. wrote the manuscript. All the authors critically reviewed the manuscript and approved the final version.

\section{Competing interests}

F.P. is a current employee of Merck Research Laboratories and A.V. is a current employee of Bristol Myers Squibb. All other authors declare no competing interests. 


\section{Additional information}

Supplementary information The online version contains supplementary material available at https://doi.org/10.1038/s41467-021-24841-y.

Correspondence and requests for materials should be addressed to A.H.B.

Peer review information Nature Communications thanks the anonymous reviewers for their contribution to the peer review of this work.

Reprints and permission information is available at http://www.nature.com/reprints

Publisher's note Springer Nature remains neutral with regard to jurisdictional claims in published maps and institutional affiliations. (c) (P) Open Access This article is licensed under a Creative Commons Attribution 4.0 International License, which permits use, sharing, adaptation, distribution and reproduction in any medium or format, as long as you give appropriate credit to the original author(s) and the source, provide a link to the Creative Commons license, and indicate if changes were made. The images or other third party material in this article are included in the article's Creative Commons license, unless indicated otherwise in a credit line to the material. If material is not included in the article's Creative Commons license and your intended use is not permitted by statutory regulation or exceeds the permitted use, you will need to obtain permission directly from the copyright holder. To view a copy of this license, visit http://creativecommons.org/licenses/by/4.0/.

(C) The Author(s) 2021 